\title{
Isomeric scandium-organic frameworks with high hydrolytic stability and selective adsorption of acetylene
}

Marina O. Barsukova, ${ }^{*}+, \neq$ Konstantin A. Kovalenko, ${ }^{\dagger}$ Anton S. Nizovtsev, ${ }^{\dagger}$ Aleksandr A. Sapianik, ${ }^{+,}+$Denis G. Samsonenko, ${ }^{+}$Danil N. Dybtsev, ${ }^{\dagger}$ and Vladimir P. Fedin ${ }^{+}$ + Nikolaev Institute of Inorganic Chemistry, Siberian Branch of the Russian Academy of Sciences, 630090, Novosibirsk, Russia

₹ Functional Materials, Design, Discovery \& Development (FMD3), Advanced Membrane \& Porous Materials Center, King Abdullah University of Science and Technology, 23955-6900, Thuwal, Kingdom of Saudi Arabia

Synthesis conditions $\quad 2$

Characterization of obtained compounds $\quad 3$

Crystallographic data $\quad 6$

$\begin{array}{ll}\text { Sorption of gases } & 7\end{array}$

Calculations of selectivity factors and heats of adsorption 11

$\begin{array}{ll}\text { DFT calculations } & 24\end{array}$

$\begin{array}{ll}\text { Iodine adsorption } & \mathbf{3 0}\end{array}$ 


\section{Synthesis conditions}

Table S1. Synthesis conditions for $1 \cdot \mathrm{DMF} \cdot 2 \mathrm{H}_{2} \mathrm{O}$ and $2 \cdot \mathrm{DMA} \cdot 4 \mathrm{H}_{2} \mathrm{O}$

\begin{tabular}{|c|c|c|c|}
\hline $\mathrm{Sc}^{3+}: \mathrm{H}_{2}$ pzc ratio & Solvent mixture & Acid & Obtained framework \\
\hline $1: 2$ & DMF, $\mathrm{H}_{2} \mathrm{O}, \mathrm{THF}$ & + & $\mathbf{1}$ \\
\hline $1: 2$ & $\mathrm{DMF}, \mathrm{H}_{2} \mathrm{O}, \mathrm{MeOH}$ & + & $\mathbf{1}$ \\
\hline $1: 2$ & $\mathrm{DMF}, \mathrm{H}_{2} \mathrm{O}, \mathrm{EtOH}$ & + & $\mathbf{1}$ \\
\hline $1: 2$ & DMF, $\mathrm{H}_{2} \mathrm{O}$ & + & $\mathbf{1}$ \\
\hline $1: 2$ & DMF, $\mathrm{H}_{2} \mathrm{O}, \mathrm{NMP}$ & + & $\mathbf{1}$ \\
\hline $1: 2$ & DMA, $\mathrm{H}_{2} \mathrm{O}, \mathrm{NMP}$ & + & $\mathbf{2}$ \\
\hline $1: 2$ & DMA, $\mathrm{H}_{2} \mathrm{O}, \mathrm{DMF}$ & + & $\mathbf{2}$ \\
\hline
\end{tabular}




\section{Characterization of obtained compounds}
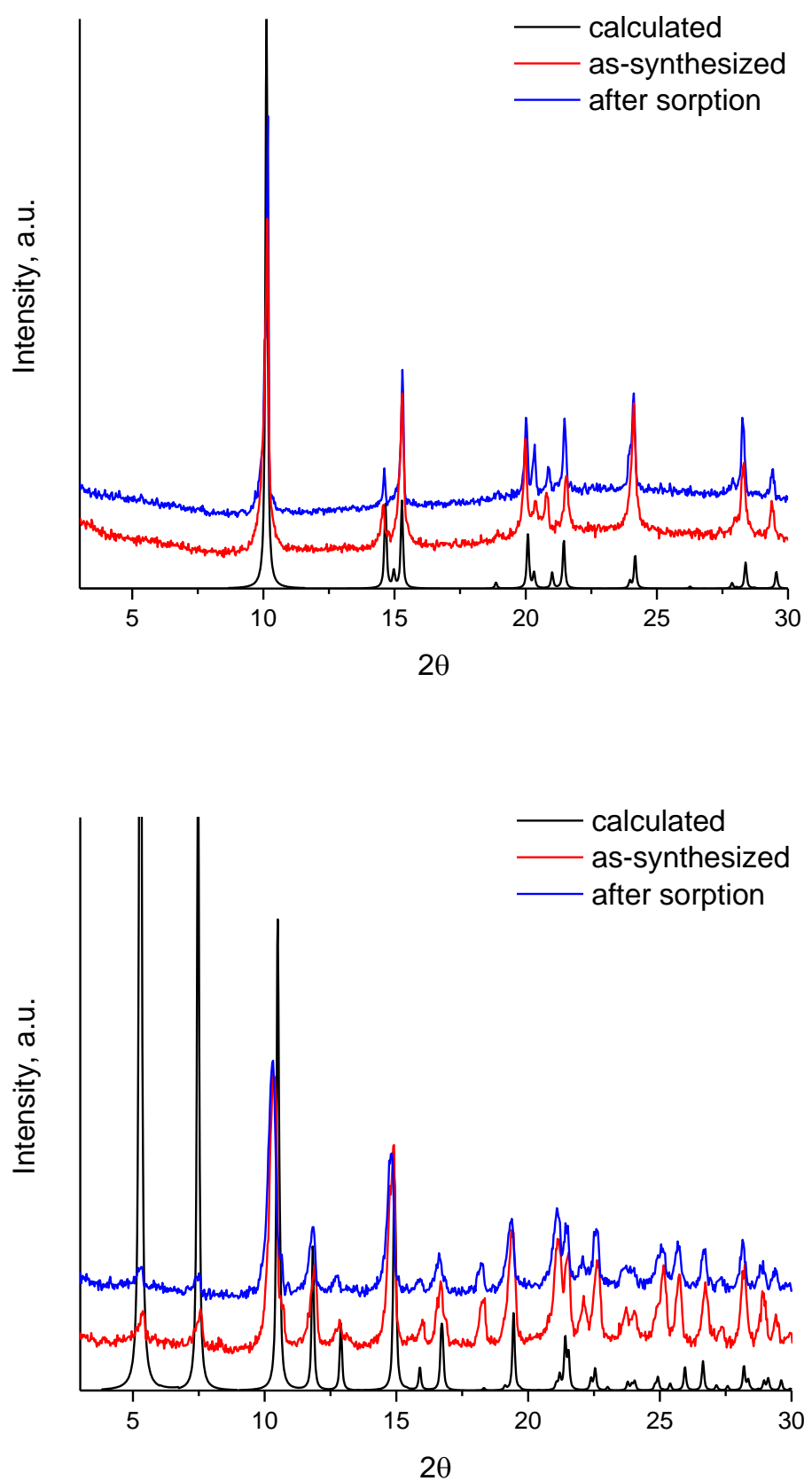

Fig. S1. PXRD patterns for as-synthesized, activated and after sorption (including water vapors) samples of 1·DMF $\cdot 2 \mathrm{H}_{2} \mathrm{O}$ (upper) and $2 \cdot \mathrm{DMA} \cdot 4 \mathrm{H}_{2} \mathrm{O}$ (bottom). 

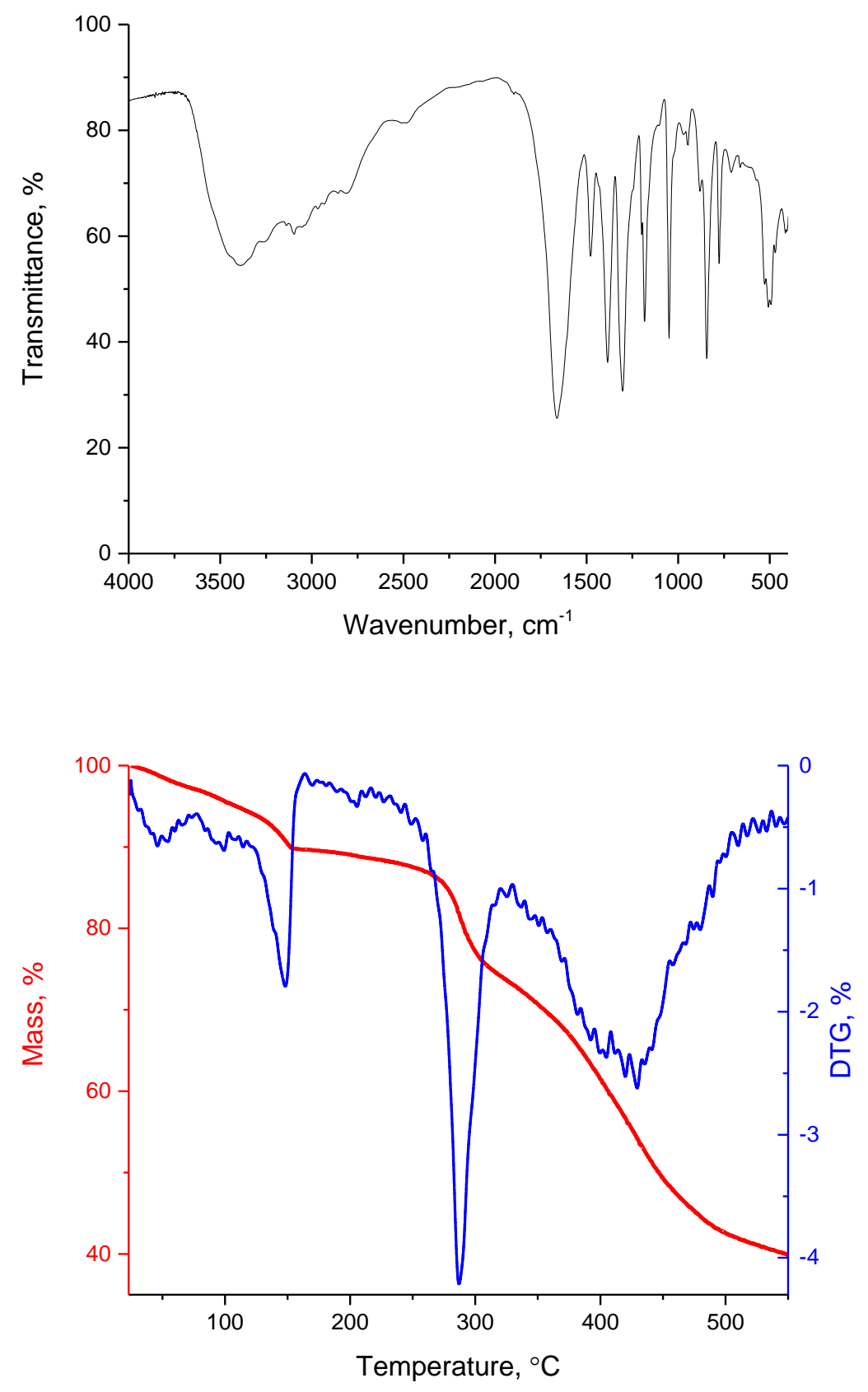

Fig. S2. IR spectrum (upper) and TG curves (bottom) for $1 \cdot \mathrm{DMF} \cdot \mathbf{2} \mathrm{H}_{2} \mathrm{O}$. 

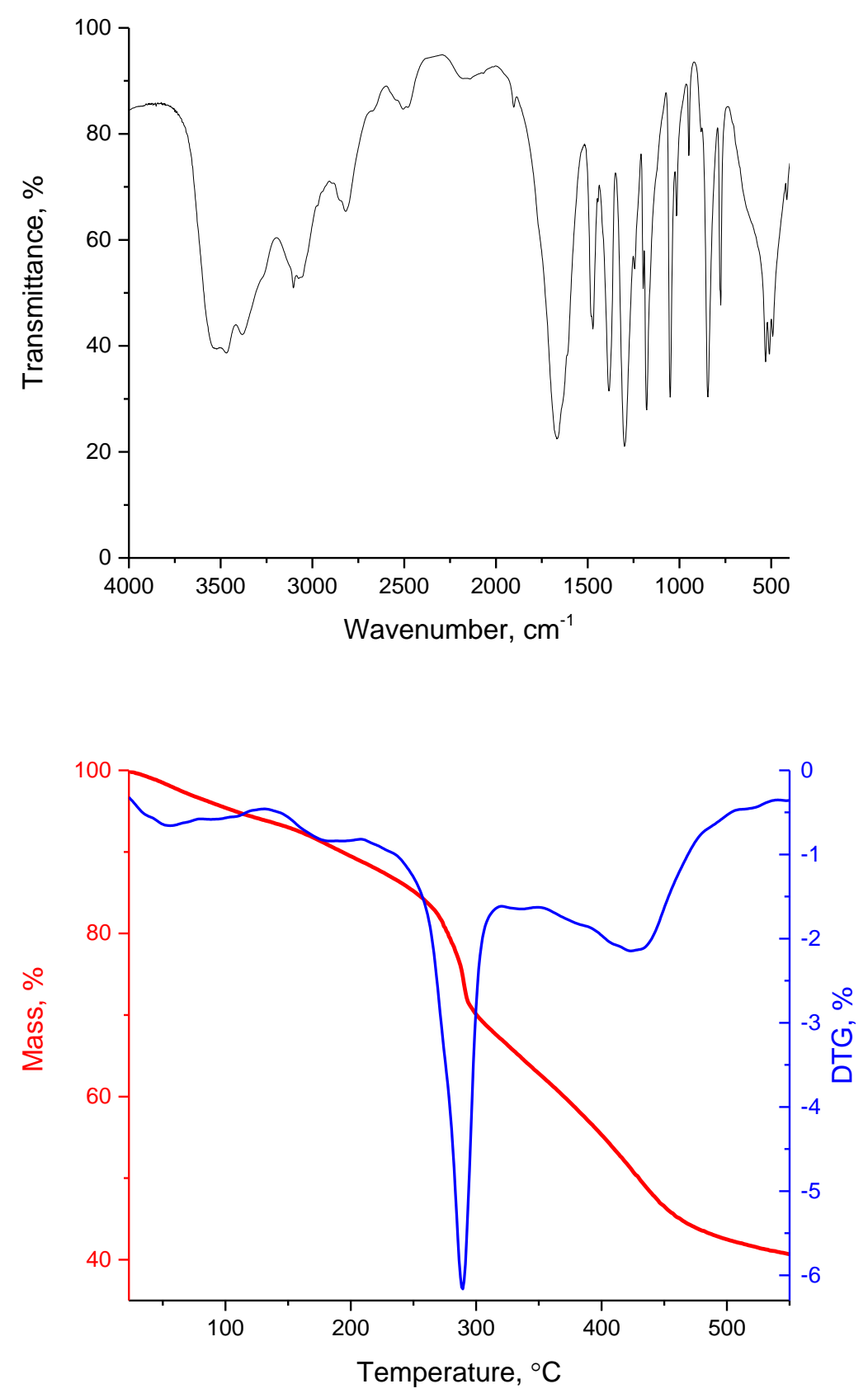

Fig. S3. IR spectrum (upper) and TG curves (bottom) for $2 \cdot \mathrm{DMA} \cdot 4 \mathrm{H}_{2} \mathrm{O}$. 


\section{Crystallographic data}

Table S2. Crystallographic data for obtained samples.

\begin{tabular}{|c|c|c|c|c|}
\hline \multirow[t]{2}{*}{ Identification code } & \multirow[t]{2}{*}{$1 \cdot \mathrm{DMF} \cdot \mathbf{2} \mathrm{H}_{2} \mathrm{O}$} & \multirow[t]{2}{*}{ 2.DMA } & \multicolumn{2}{|c|}{$2 \cdot \mathbf{I}_{2} \cdot 2 \mathrm{H}_{2} \mathrm{O}$} \\
\hline & & & $\begin{array}{c}\text { Before } \\
\text { SQUEEZE }\end{array}$ & After SQUEEZE \\
\hline Formula & $\mathrm{C}_{15} \mathrm{H}_{16} \mathrm{~N}_{5} \mathrm{O}_{11} \mathrm{Sc}$ & $\mathrm{C}_{16} \mathrm{H}_{14} \mathrm{~N}_{5} \mathrm{O}_{9} \mathrm{Sc}$ & $\mathrm{C}_{12} \mathrm{H}_{9} \mathrm{I}_{1.8} \mathrm{~N}_{4} \mathrm{O}_{10} \mathrm{Sc}$ & $\mathrm{C}_{12} \mathrm{H}_{9} \mathrm{I}_{2} \mathrm{~N}_{4} \mathrm{O}_{10} \mathrm{Sc}$ \\
\hline Radiation wavelength, $\AA$ & 0.71073 & 0.71073 & 0.79313 & 0.79313 \\
\hline $\begin{array}{l}\text { Measurement } \\
\text { temperature, } K\end{array}$ & 130 & 130 & 100 & 100 \\
\hline$M, \mathrm{~g} / \mathrm{mol}$ & 487.29 & 465.28 & 667.99 & 667.99 \\
\hline Crystal system & tetragonal & tetragonal & tetragonal & tetragonal \\
\hline Space group & I $4_{1} / a$ & $\mathrm{P} 4 / n n c$ & $\mathrm{P} 4 / n n c$ & $\mathrm{P} 4 / n n c$ \\
\hline$a, \AA$ & $9.3466(4)$ & $23.6420(4)$ & $23.400(3)$ & $23.400(3)$ \\
\hline$b, \AA$ & $9.3466(4)$ & $23.6420(4)$ & $23.400(3)$ & $23.400(3)$ \\
\hline$c, \AA$ & 23.7893(14) & $9.0156(3)$ & $9.460(9)$ & $9.460(9)$ \\
\hline$V, \AA^{3}$ & $2078.2(2)$ & $5039.2(2)$ & $5180(5)$ & $5180(5)$ \\
\hline$Z$ & 4 & 8 & 8 & 8 \\
\hline$D($ calcd $), \mathrm{g} / \mathrm{cm}^{3}$ & 1.557 & 1.227 & 1.713 & 1.713 \\
\hline$\mu, \mathrm{mm}^{-1}$ & 0.423 & 0.340 & 3.604 & 3.604 \\
\hline$F(000)$ & 1000 & 1904 & 2528 & 2528 \\
\hline Crystal size, $\mathrm{mm}$ & $\begin{array}{c}0.28 \times 0.18 \times \\
0.17\end{array}$ & $0.53 \times 0.34 \times 0.25$ & $0.10 \times 0.10 \times 0.05$ & $0.10 \times 0.10 \times 0.05$ \\
\hline $\begin{array}{l}\theta \text { range for data } \\
\text { collection, deg. }\end{array}$ & $3.369-29.307$ & $3.434-29.450$ & $1.373-28.527$ & $1.373-28.527$ \\
\hline Index ranges & $\begin{array}{l}-9 \leq \mathrm{h} \leq 11 \\
-12 \leq \mathrm{k} \leq 8 \\
-31 \leq 1 \leq 17\end{array}$ & $\begin{array}{c}-29 \leq \mathrm{h} \leq 18 \\
-32 \leq \mathrm{k} \leq 21 \\
-12 \leq 1 \leq 6\end{array}$ & $\begin{aligned}-23 & \leq \mathrm{h} \leq 28, \\
-23 & \leq \mathrm{k} \leq 28 \\
-11 & \leq 1 \leq 11\end{aligned}$ & $\begin{aligned}-23 & \leq \mathrm{h} \leq 28 \\
-23 & \leq \mathrm{k} \leq 28 \\
-11 & \leq 1 \leq 11\end{aligned}$ \\
\hline $\begin{array}{c}\text { Reflections } \\
\text { collected/independent }\end{array}$ & $3000 / 1249$ & $13202 / 3116$ & $9680 / 2358$ & $9680 / 2352$ \\
\hline Reflections with $I>2 \sigma(I)$ & 1141 & 2427 & 1645 & 1639 \\
\hline$R_{\text {int }}$ & 0.0129 & 0.0176 & 0.0487 & 0.0483 \\
\hline GOF on $F^{2}$ & 1.108 & 1.085 & 1.123 & 1.125 \\
\hline$R_{1}, w R_{2}[I>2 \sigma(I)]$ & $0.0280,0.0742$ & $0.0442,0.1430$ & $0.1198,0.3944$ & $0.1102,0.3644$ \\
\hline$R_{1}, w R_{2}$ (all data) & $0.0311,0.0754$ & $0.0568,0.1500$ & $0.1529,0.4211$ & $0.1324,0.3839$ \\
\hline$\Delta \rho_{\max } / \Delta \rho_{\min ,}, \mathrm{e} \AA^{-3}$ & $0.347 /-0.197$ & $0.490 /-0.274$ & $0.894 /-0.675$ & $1.228 /-0.398$ \\
\hline
\end{tabular}




\section{Sorption of gases}
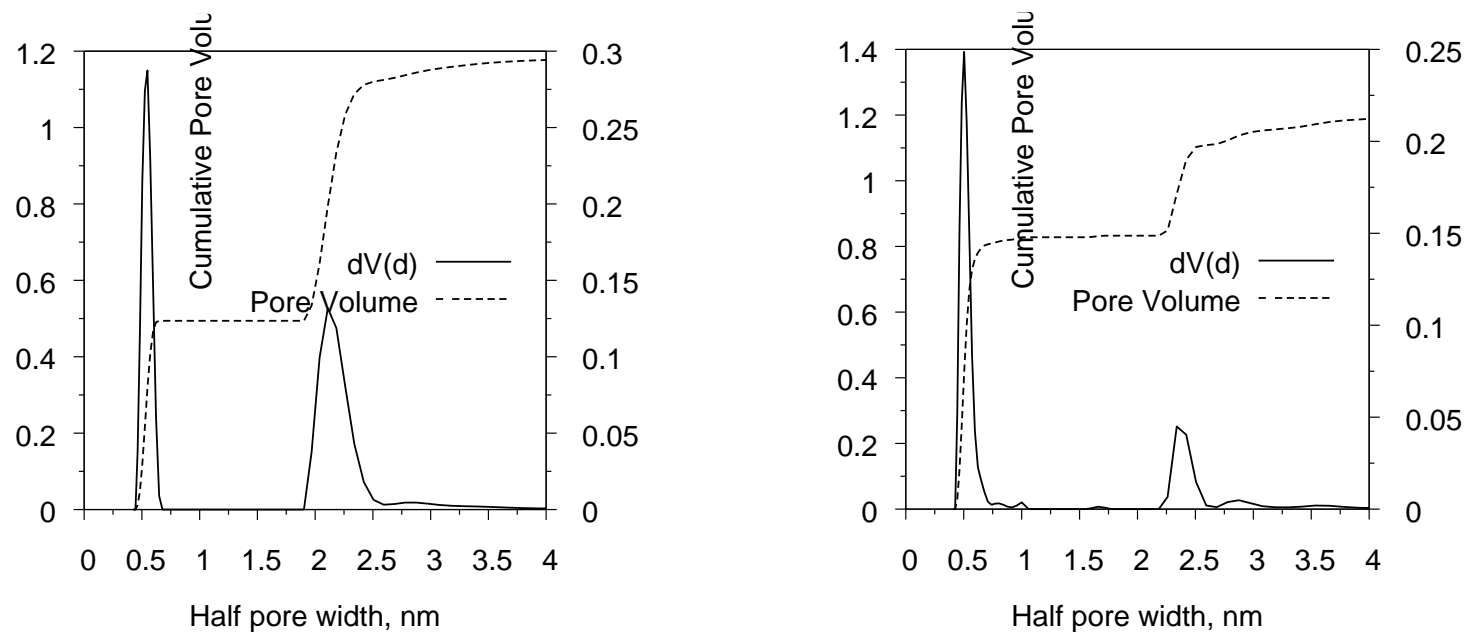

Fig. S4. Pore size distribution for 1 (left) and 2 (right) according to DFT calculations.

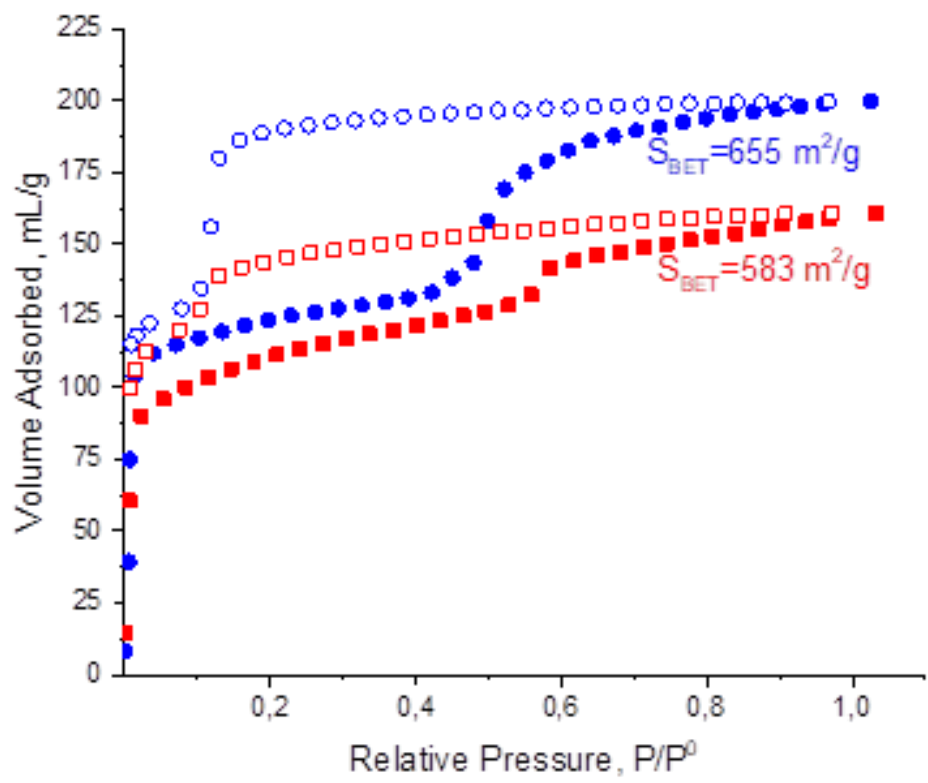

Fig. S5. $\mathrm{CO}_{2}$ sorption isotherms with calculated BET surfaces after water vapor sorption for 1 (blue) and 2 (red). 

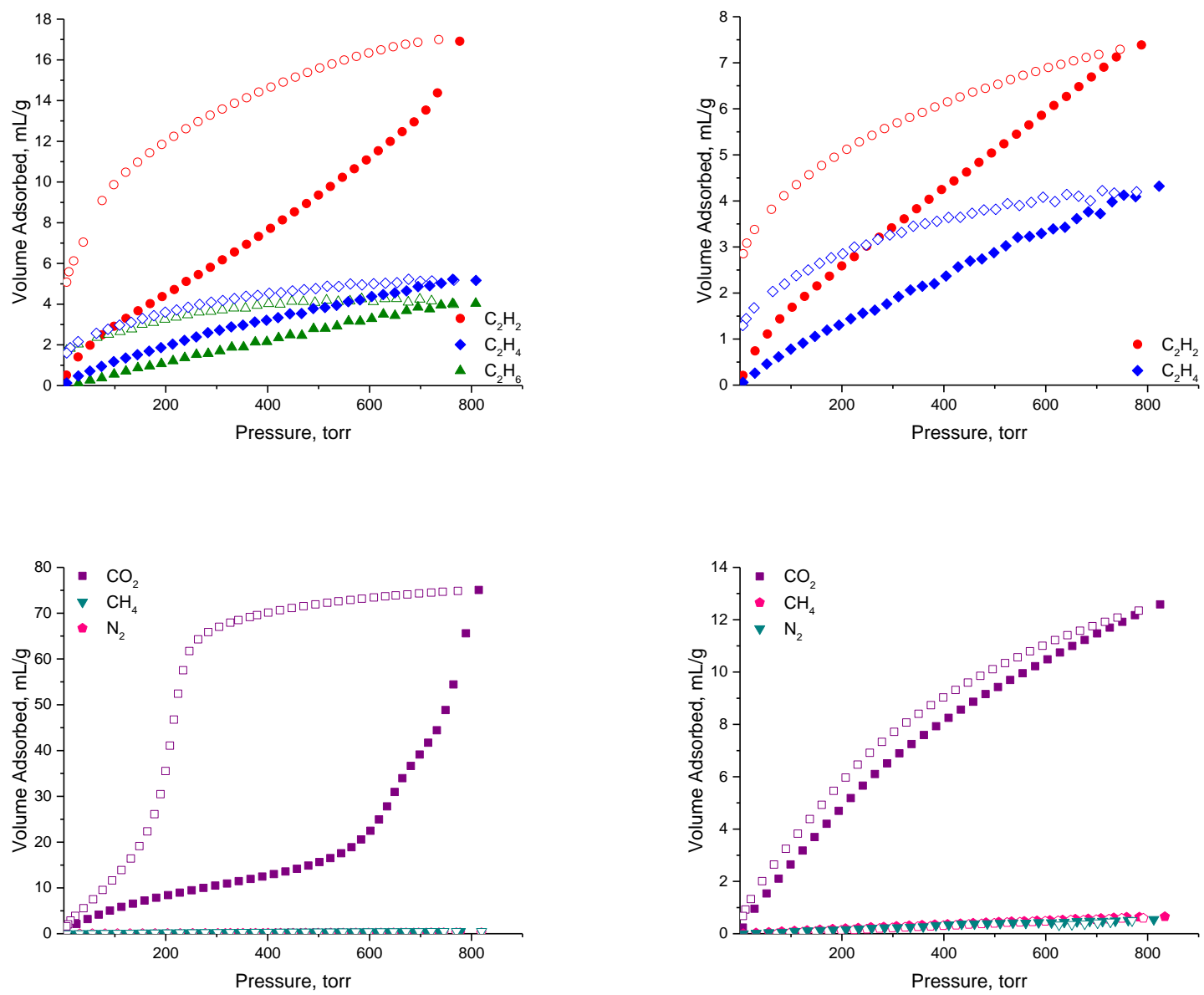

Fig. S6. Gas isotherms for 1 at 273 K (left) and 298 K (right). 

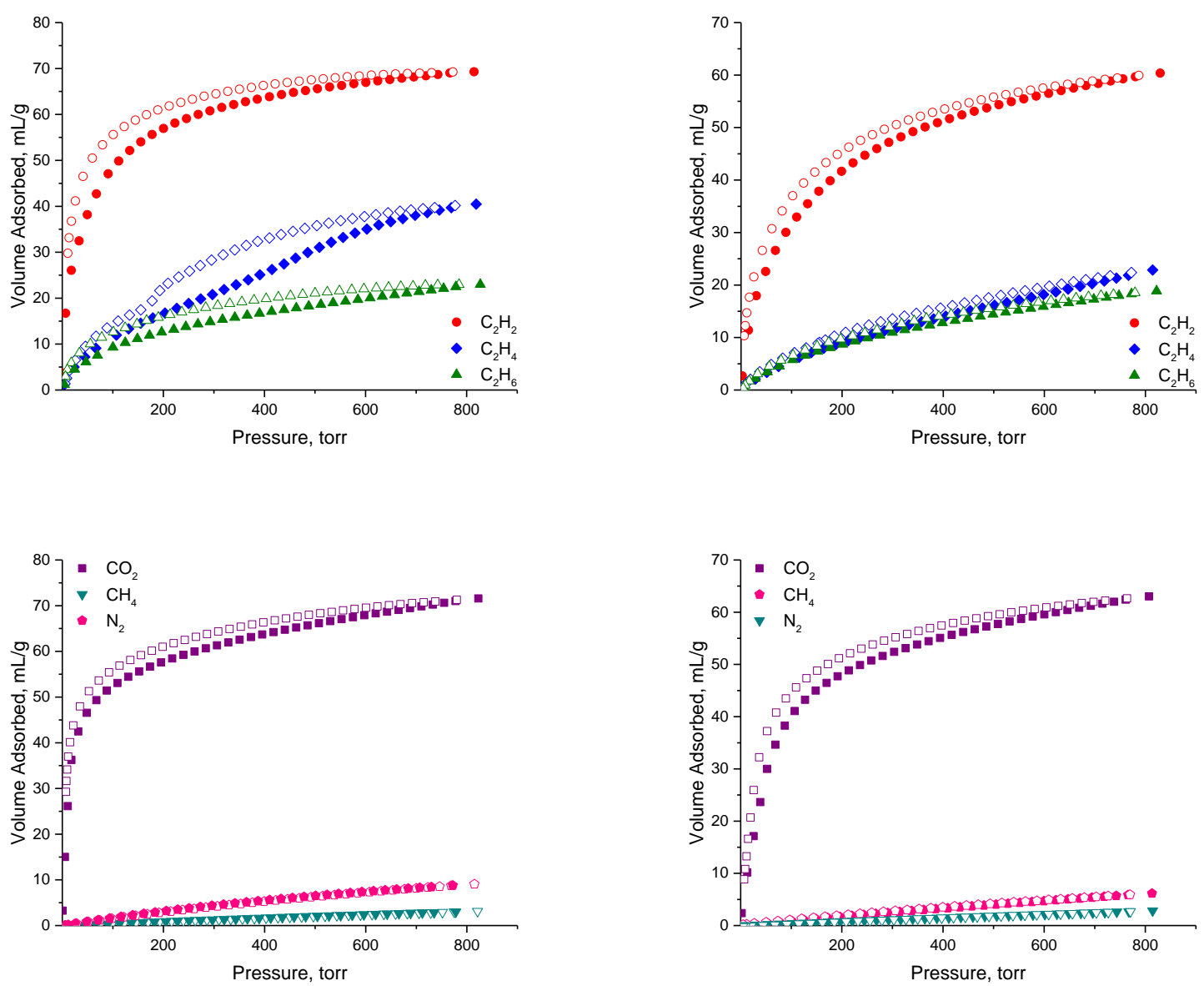

Fig. S7. Gas isotherms for 2 at 273 K (left) and 298 K (right). 
Table S3. Gas uptakes at 1 bar and $273 \mathrm{~K}$ and $298 \mathrm{~K}$ in different units.

\begin{tabular}{|c|c|c|c|c|c|c|}
\hline \multirow{2}{*}{ Gas } & \multicolumn{3}{|c|}{$273 \mathrm{~K}$} & \multicolumn{3}{|c|}{$298 \mathrm{~K}$} \\
\hline & $\mathrm{mL}(\mathrm{STP}) / \mathrm{g}$ & $\mathrm{mmol} / \mathrm{g}$ & wt. $\%$ & mL(STP)/g & $\mathrm{mmol} / \mathrm{g}$ & wt. $\%$ \\
\hline \multicolumn{7}{|c|}{1} \\
\hline $\mathrm{C}_{2} \mathrm{H}_{2}$ & 14.9 & 0.665 & $1.7 \%$ & 7.3 & 0.326 & $0.9 \%$ \\
\hline $\mathrm{C}_{2} \mathrm{H}_{4}$ & 5.1 & 0.228 & $0.6 \%$ & 4.1 & 0.183 & $0.5 \%$ \\
\hline $\mathrm{C}_{2} \mathrm{H}_{6}$ & 4 & 0.178 & $0.5 \%$ & - & - & - \\
\hline $\mathrm{CO}_{2}$ & 48.9 & 2.182 & $9.6 \%$ & 11.9 & 0.531 & $2.3 \%$ \\
\hline $\mathrm{CH}_{4}$ & $<0.6$ & $<0.027$ & $<0.04 \%$ & $<0.6$ & $<0.027$ & $<0.04 \%$ \\
\hline $\mathrm{N}_{2}$ & $<0.5$ & $<0.022$ & $<0.06 \%$ & $<0.5$ & $<0.022$ & $<0.06 \%$ \\
\hline \multicolumn{7}{|c|}{2} \\
\hline $\mathrm{C}_{2} \mathrm{H}_{2}$ & 69.3 & 3.09 & 8.0 & 60.4 & 2.70 & 7.0 \\
\hline $\mathrm{C}_{2} \mathrm{H}_{4}$ & 40.5 & 1.81 & 5.1 & 22.9 & 1.02 & 2.9 \\
\hline $\mathrm{C}_{2} \mathrm{H}_{6}$ & 23.0 & 1.03 & 3.1 & 18.9 & 0.84 & 2.5 \\
\hline $\mathrm{CO}_{2}$ & 71.6 & 3.20 & 14.1 & 63.6 & 2.84 & 12.5 \\
\hline $\mathrm{CH}_{4}$ & 9.1 & 0.41 & 0.7 & 6.2 & 0.28 & 0.4 \\
\hline $\mathrm{N}_{2}$ & 3.1 & 0.14 & 0.4 & 2.8 & 0.12 & 0.4 \\
\hline
\end{tabular}




\section{Calculations of selectivity factors and heats of adsorption}

\section{Zero coverage isosteric heats of adsorption}

Isosteric heats of adsorption were calculated using virial coefficients (Table S4) by equation (S1).

$$
\Delta H^{\circ}=R \cdot \sum_{i} A_{i} \cdot n^{i}
$$

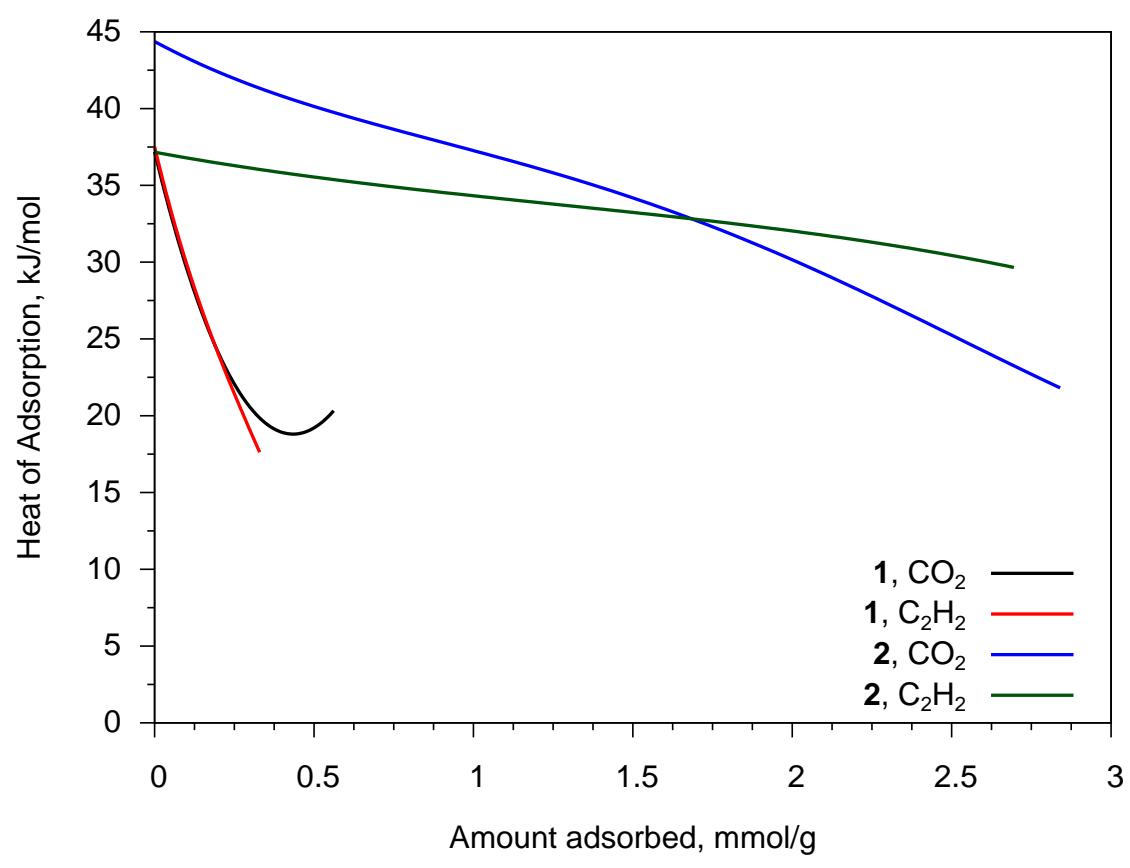

Fig. S8. The heats of adsorption curves for frameworks 1 and 2.

\section{Isotherms fit by virial equation}

Gas adsorption isotherms at $273 \mathrm{~K}$ and $298 \mathrm{~K}$ were fitted by virial equation (S2) in order to calculate Henry constants and isosteric heats of adsorption.

$$
\ln p=\ln n+\frac{1}{T} \sum_{i} A_{i} \cdot n^{i}+\sum_{j} B_{j} \cdot n^{j}
$$

Virial coefficients are summarized in Table S4, whereas fit plot are shown in Fig. S9-S11. 
Table S4. Virial coefficients $A_{i}$ and $B_{i}$ for gas adsorption isotherms at $273 \mathrm{~K}$ and $298 \mathrm{~K}$ on $\mathbf{1}$ and 2.

\begin{tabular}{|c|c|c|}
\hline \multirow{2}{*}{ Gas } & \multicolumn{2}{|c|}{ Coefficients } \\
\hline & 1 & 2 \\
\hline $\mathrm{C}_{2} \mathrm{H}_{2}$ & $\begin{array}{l}A_{0}=-4512.36 ; A_{1}=10553.6 \\
A_{2}=-16296.4 ; A_{3}=23117 \\
A_{4}=-12056.5 \\
B_{0}=14.828 ; B_{1}=-20.2167\end{array}$ & $\begin{array}{c}A_{0}=-4469.24 ; A_{1}=457.027 ; \\
A_{2}=-157.657 ; A_{3}=41.6724 ; \\
B_{0}=11.1162\end{array}$ \\
\hline $\mathrm{C}_{2} \mathrm{H}_{4}$ & $\begin{array}{c}A_{0}=-2331.21 ; A_{1}=8941.12 \\
A_{2}=-5921.8 \\
B_{0}=8.95599 ; B_{1}=-23.2633\end{array}$ & $\begin{array}{c}A_{0}=-3601.61 ; A_{1}=788.545 \\
A_{2}=-499.176 ; A_{3}=119.063 ; \\
B_{0}=10.8315\end{array}$ \\
\hline $\mathrm{C}_{2} \mathrm{H}_{6}$ & $-^{\mathrm{a}}$ & $\begin{array}{l}A_{0}=-3895.83 ; A_{1}=3160.18 ; \\
A_{2}=-272.914 ; \\
B_{0}=11.8922 ; B_{1}=-8.13684\end{array}$ \\
\hline $\mathrm{CO}_{2}$ & $\begin{array}{c}A_{0}=-4472.40 ; A_{1}=10315.2 \\
A_{2}=-12374.5 ; A_{3}=855.5 \\
A_{4}=-136.8 \\
B_{0}=14.807 ; B_{1}=-32.1994 \\
B_{2}=38.4903\end{array}$ & $\begin{array}{c}A_{0}=-5336.08 ; A_{1}=1353.82 ; \\
A_{2}=-880.113 ; A_{3}=445.721 ; \\
A_{4}=-65.2367 ; \\
B_{0}=14.05 ; B_{1}=-2.15874\end{array}$ \\
\hline $\mathrm{CH}_{4}$ & $-^{\mathrm{a}}$ & $\begin{array}{c}A_{0}=-1827.51 ; A_{1}=230.941 ; \\
B_{0}=7.31384\end{array}$ \\
\hline $\mathrm{N}_{2}$ & $-^{\mathrm{a}}$ & $\begin{array}{c}A_{0}=-394.455 ; A_{1}=256.232 ; \\
B_{0}=3.34947\end{array}$ \\
\hline
\end{tabular}

${ }^{a}$ low adsorption capacity 
Table S5. Henry constants for gas adsorption on $\mathbf{1}$ and $\mathbf{2}$ in $\mathrm{mmol} \cdot \mathrm{g}^{-1} \cdot \mathrm{bar}^{-1}$ at $273 \mathrm{~K}$ and $298 \mathrm{~K}$.

\begin{tabular}{|c|c|c|c|c|}
\hline \multirow{2}{*}{ Gas } & \multicolumn{2}{|c|}{1} & \multicolumn{2}{c|}{} \\
\cline { 2 - 5 } & $\mathbf{2 7 3 ~ K}$ & $\mathbf{2 9 8} \mathbf{K}$ & $\mathbf{2 7 3 ~ K}$ & $\mathbf{2 9 8 ~ K}$ \\
\hline $\mathrm{C}_{2} \mathrm{H}_{2}$ & 5.429 & 1.359 & 189.8 & 48.12 \\
\hline $\mathrm{C}_{2} \mathrm{H}_{4}$ & 0.6561 & 0.3208 & 10.53 & 3.485 \\
\hline $\mathrm{C}_{2} \mathrm{H}_{6}$ & $-^{\mathrm{a}}$ & $-^{\mathrm{a}}$ & 10.70 & 3.236 \\
\hline $\mathrm{CO}_{2}$ & 4.788 & 1.213 & 241.1 & 46.87 \\
\hline $\mathrm{CH}_{4}$ & $-^{\mathrm{a}}$ & $-^{\mathrm{a}}$ & 0.5361 & 0.3059 \\
\hline $\mathrm{N}_{2}$ & $-^{\mathrm{a}}$ & $-^{\mathrm{a}}$ & 0.1488 & 0.1318 \\
\hline
\end{tabular}

${ }^{\text {a }}$ low adsorption capacity 

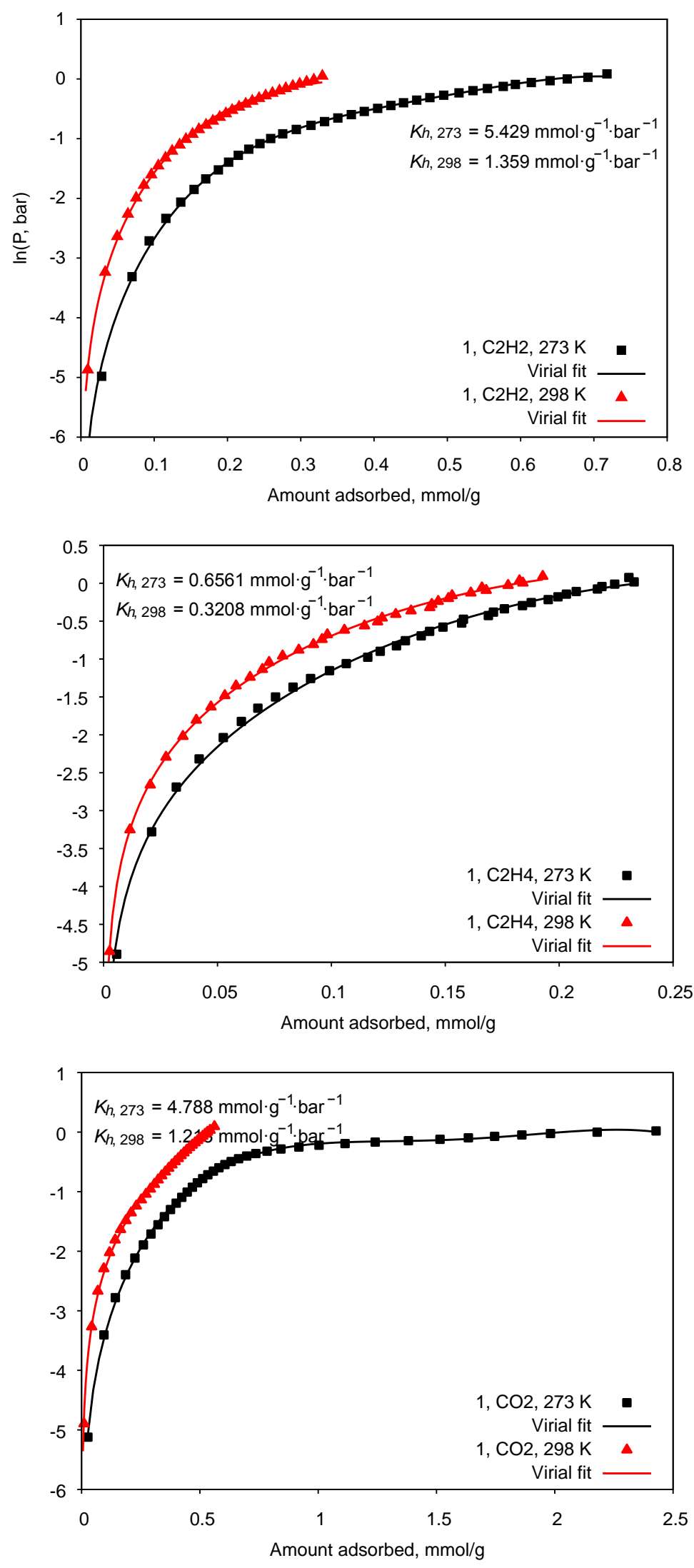

Fig. S9. Fits of $\mathrm{C}_{2} \mathrm{H}_{2}, \mathrm{C}_{2} \mathrm{H}_{4}$ and $\mathrm{CO}_{2}$ isotherms by virial equations for $\mathbf{1}$. 

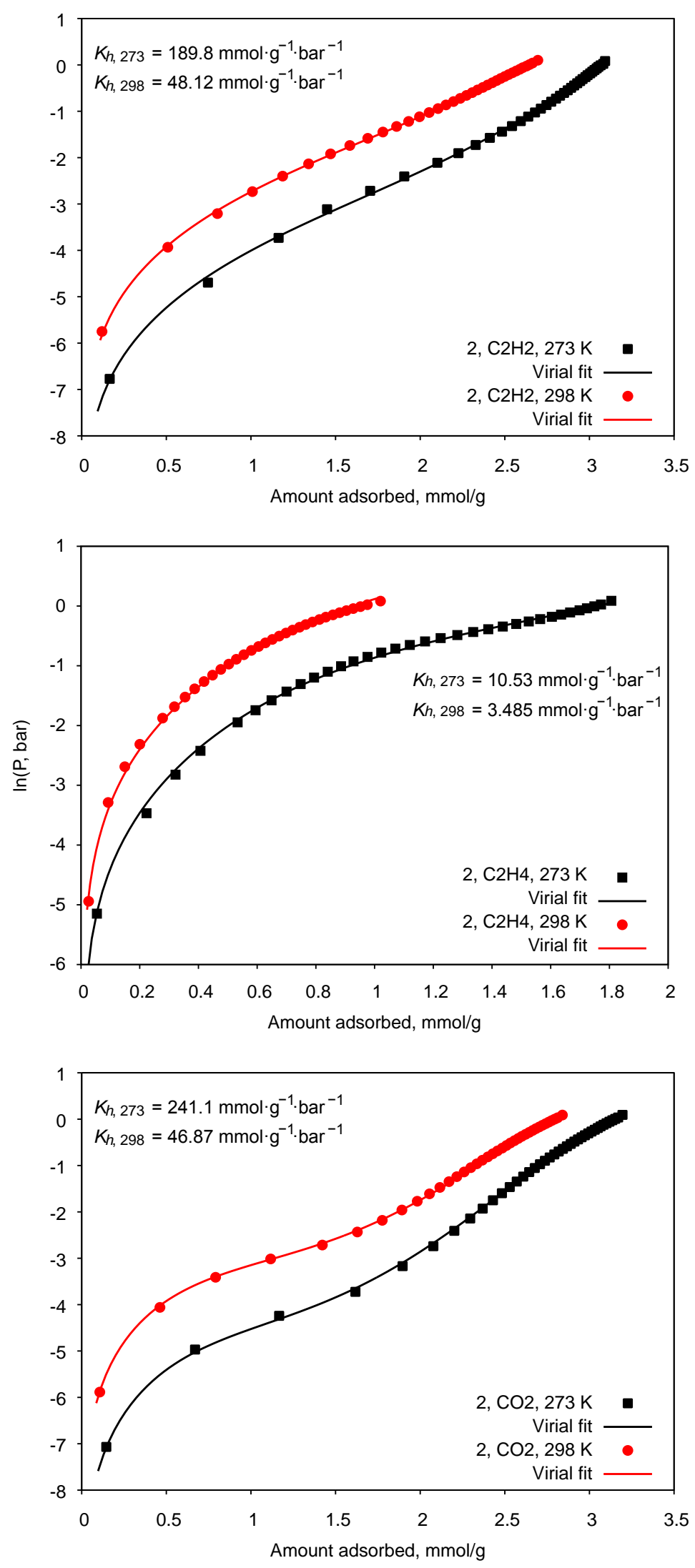

Fig. S10. Fits of $\mathrm{C}_{2} \mathrm{H}_{2}, \mathrm{C}_{2} \mathrm{H}_{4}$ and $\mathrm{CO}_{2}$ isotherms by virial equations for 2 . 

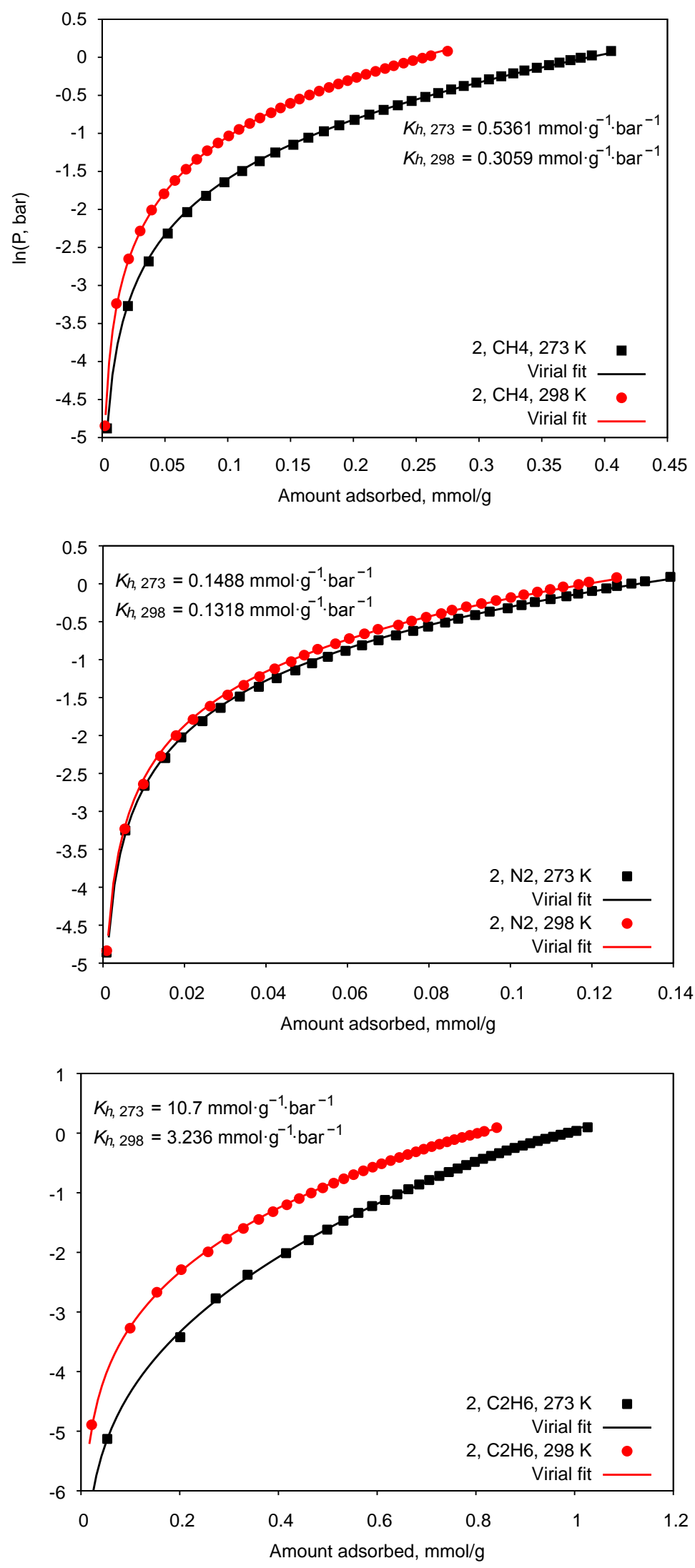

Fig. S11. Fits of $\mathrm{CH}_{4}, \mathrm{~N}_{2}$ and $\mathrm{C}_{2} \mathrm{H}_{6}$ isotherms by virial equations for 2 . 


\section{Fit of adsorption isotherms}

Adsorption isotherm for all studied gases on 1 were fitted by power function due to it's complex curvature:

$$
n=\sum_{i} A_{i} \cdot p^{i}
$$

Adsorption isotherms were fitted by the most appropriate equation for IAST calculations. Fittings were performed for isotherms in $\mathrm{mL} / \mathrm{g}$-torr units, so parameters are in the corresponding units. The list of equations for 2 sample:

- $\mathrm{C}_{2} \mathrm{H}_{2}$ : Langmuir-Freundlich model $n[\mathrm{~mL} / \mathrm{g}]=\frac{w b p[\text { torr }]^{1 / t}}{1+b p[\text { torr }]^{1 / t}}$

- $\mathrm{C}_{2} \mathrm{H}_{4}$ : Dual-Site Langmuir-Freundlich model at $273 \mathrm{~K} n=\frac{w_{1} b_{1} p^{1 / t_{1}}}{1+b_{1} p^{1 / t_{1}}}+\frac{w_{2} b_{2} p^{1 / t_{2}}}{1+b_{2} p^{1 / t_{2}}}$

- and Sum of Henry and Langmuir-Freundlich model at $298 \mathrm{~K} n[\mathrm{~mL} / \mathrm{g}]=K_{H} \cdot p[$ torr $]+$ $\frac{w b p[\text { torr }]^{1 / t}}{1+b p[\text { torr }]^{1 / t}}$

- $\mathrm{C}_{2} \mathrm{H}_{6}$ : Sum of Henry and Langmuir-Freundlich model

- $\mathrm{CO}_{2}$ : Sum of Henry and Langmuir-Freundlich model

- $\mathrm{CH}_{4}$ : Langmuir model $n[\mathrm{~mL} / \mathrm{g}]=\frac{w b p[\text { torr }]}{1+b p[\text { torr }]}$

- $\mathrm{N}_{2}$ : Langmuir model

Fitted parameters are summarized in Table S6. Fitted isotherms are shown in Fig. S12. 
Table S6. Fitted parameters for adsorption isotherms at $273 \mathrm{~K}$ and $298 \mathrm{~K}$

\begin{tabular}{|c|c|c|c|c|}
\hline \multirow[b]{2}{*}{ Gas } & \multicolumn{2}{|c|}{1} & \multicolumn{2}{|c|}{2} \\
\hline & $273 \mathrm{~K}$ & $298 \mathrm{~K}$ & $273 \mathrm{~K}$ & $298 \mathrm{~K}$ \\
\hline $\mathrm{C}_{2} \mathrm{H}_{2}$ & $\begin{array}{c}\mathrm{A}_{1}=0.04918 \\
\mathrm{~A}_{2}=-0.0002318 \\
\mathrm{~A}_{3}=6.844 \mathrm{e}-07 \\
\mathrm{~A}_{4}=-8.912 \mathrm{e}-10 \\
\mathrm{~A}_{5}=4.284 \mathrm{e}-13\end{array}$ & $\begin{array}{c}\mathrm{A}_{1}=0.02394 \\
\mathrm{~A}_{2}=-9.405 \mathrm{e}-05 \\
\mathrm{~A}_{3}=2.578 \mathrm{e}-07 \\
\mathrm{~A}_{4}=-3.265 \mathrm{e}-10 \\
\mathrm{~A}_{5}=1.550 \mathrm{e}-13\end{array}$ & $\begin{array}{c}w=8.249 \\
b=0.06867 \\
t=1.478\end{array}$ & $\begin{array}{c}w=7.966 \\
b=0.02787 \\
t=1.380\end{array}$ \\
\hline $\mathrm{C}_{2} \mathrm{H}_{4}$ & $\begin{array}{c}\mathrm{A}_{1}=0.01243 \\
\mathrm{~A}_{2}=-1.430 \mathrm{e}-05 \\
\mathrm{~A}_{3}=9.161 \mathrm{e}-09\end{array}$ & $\begin{array}{c}\mathrm{A}_{1}=0.007621 \\
\mathrm{~A}_{2}=-5.276 \mathrm{e}-06 \\
\mathrm{~A}_{3}=3.050 \mathrm{e}-09\end{array}$ & $\begin{array}{c}\mathrm{w} 1=1.657 \\
\mathrm{~b} 1=0.3300 \\
\mathrm{t} 1=0.219 \\
\mathrm{w} 2=4.595 \\
\mathrm{~b} 2=0.01127 \\
\mathrm{t} 2=1.355\end{array}$ & $\begin{array}{c}\mathrm{k}=0.01677 \\
\mathrm{w}=7.339 \\
\mathrm{~b}=0.00113 \\
\mathrm{t}=0.8958\end{array}$ \\
\hline $\mathrm{C}_{2} \mathrm{H}_{6}$ & $\mathrm{~A}_{1}=0.005414$ & 一 & $\begin{array}{c}\mathrm{k}=0.01546 \\
\mathrm{w}=1.205 \\
\mathrm{~b}=0.02786 \\
\mathrm{t}=1.269\end{array}$ & $\begin{array}{c}\mathrm{k}=0.01152 \\
\mathrm{w}=1.442 \\
\mathrm{~b}=0.00194 \\
\mathrm{t}=1.074\end{array}$ \\
\hline $\mathrm{CO}_{2}$ & $\begin{array}{c}\mathrm{A}_{1}=0.1039 \\
\mathrm{~A}_{2}=-0.0008634 \\
\mathrm{~A}_{3}=4.892 \mathrm{e}-06 \\
\mathrm{~A}_{4}=-1.451 \mathrm{e}-08 \\
\mathrm{~A}_{5}=2.179 \mathrm{e}-11 \\
\mathrm{~A}_{6}=-1.506 \mathrm{e}-14 \\
\mathrm{~A}_{7}=3.702 \mathrm{e}-18\end{array}$ & $\begin{array}{c}\mathrm{A}_{1}=0.02584 \\
\mathrm{~A}_{2}=-1.346 \mathrm{e}-05\end{array}$ & $\begin{array}{c}\mathrm{k}=0.01343 \\
\mathrm{w}=5.195 \\
\mathrm{~b}=0.07706 \\
\mathrm{t}=0.9691\end{array}$ & $\begin{array}{c}\mathrm{k}=0.0171 \\
\mathrm{w}=5.284 \\
\mathrm{~b}=0.00383 \\
\mathrm{t}=0.7333\end{array}$ \\
\hline $\mathrm{CH}_{4}$ & $\begin{array}{c}\mathrm{A}_{1}=0.001081 \\
\mathrm{~A}_{2}=-5.611 \mathrm{e}-07\end{array}$ & $\begin{array}{c}\mathrm{A}_{1}=0.0009987 \\
\mathrm{~A}_{2}=-2.403 \mathrm{e}-07\end{array}$ & $\begin{array}{c}\mathrm{w}=2.274 \\
\mathrm{~b}=0.005595\end{array}$ & $\begin{array}{c}w=3.756 \\
b=0.002295\end{array}$ \\
\hline $\mathrm{N}_{2}$ & $\mathrm{~A}_{1}=0.0003053$ & $\mathrm{~A}_{1}=0.0007254$ & $\begin{array}{c}\mathrm{w}=1.836 \\
\mathrm{~b}=0.002736\end{array}$ & $\begin{array}{c}\mathrm{w}=2.163 \\
\mathrm{~b}=0.001955\end{array}$ \\
\hline
\end{tabular}



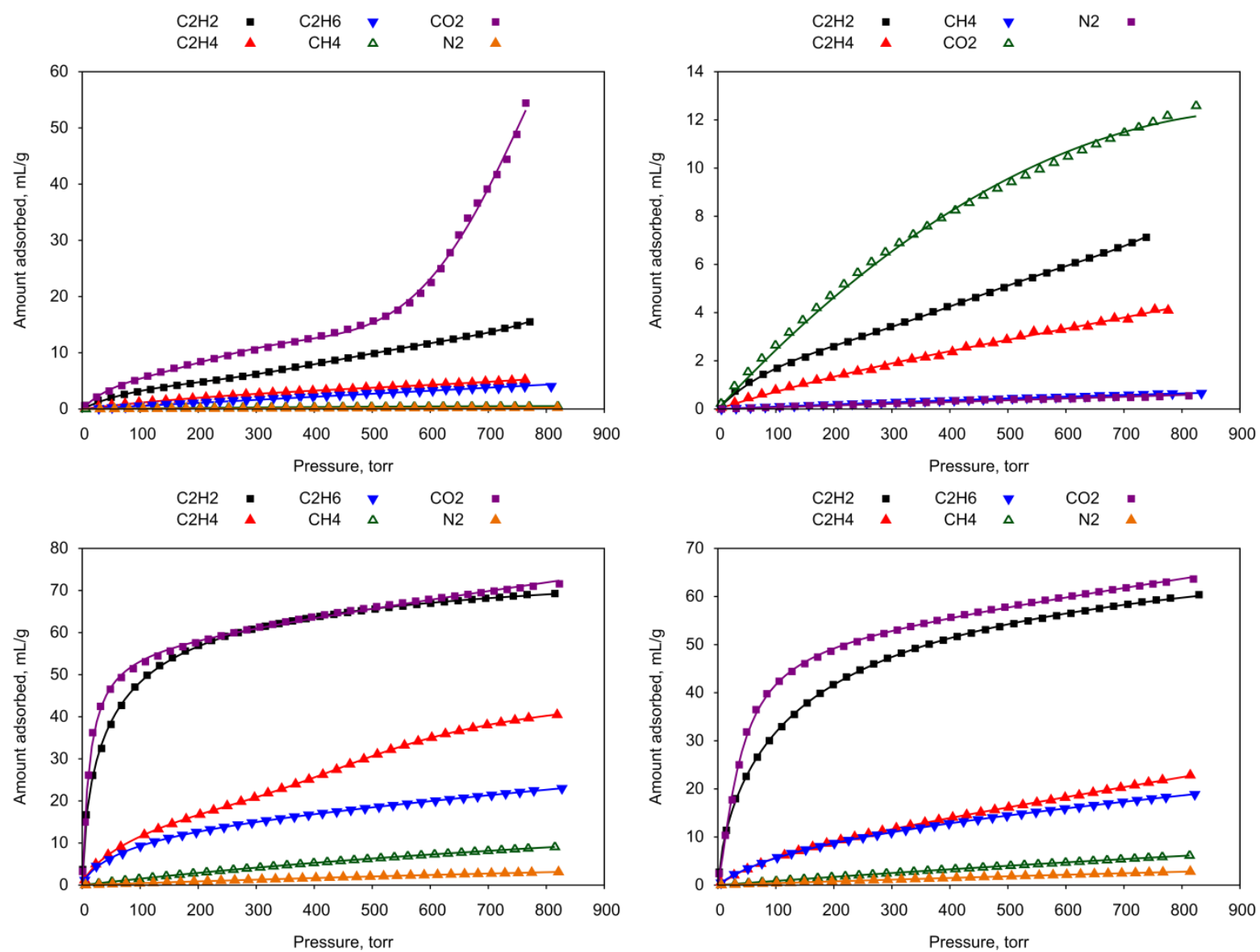

Fig. S12. Fits of isotherms of gases adsorption on 1 (upper line) and 2 (lower line) by appropriate models at $273 \mathrm{~K}$ (left column) and $298 \mathrm{~K}$ (right column). 


\section{Selectivity calculations}

Selectivity factors were evaluated by three commonly used methods:

(i) As the molar ratio of the adsorption quantities at the relevant partial pressures of the gases:

$$
S=\frac{n_{1} / n_{2}}{p_{1} / p_{2}}
$$

where $S$ is the selectivity factor, $n_{i}$ represents the adsorbed amount of component $i$, and $p_{i}$ represents the partial pressure of component $i$.

(ii) As a ratio of Henry constants which corresponds to the slope of the adsorption isotherm at very low partial pressures:

$$
S=\frac{K_{H 1}}{K_{H 2}}
$$

(iii) By ideal adsorbed solution theory (IAST). The relationship between $P, y_{i}$ and $x_{i}(P$ the total pressure of the gas phase, $y_{i}$ - mole fraction of the $i$-component in gas phase, $x_{i}$ - mole fraction of the $i$-component in absorbed state) is defined according to the IAST theory:

$$
\int_{p=0}^{p=\frac{P y_{1}}{x_{1}}} n_{1}(p) d \ln p=\int_{p=0}^{p=\frac{P y_{2}}{x_{2}}} n_{2}(p) d \ln p
$$

In this case the selectivity factors were determined as:

$$
S=\frac{y_{2} x_{2}}{y_{1} x_{1}}=\frac{x_{1}\left(1-y_{1}\right)}{y_{1}\left(1-x_{1}\right)}
$$



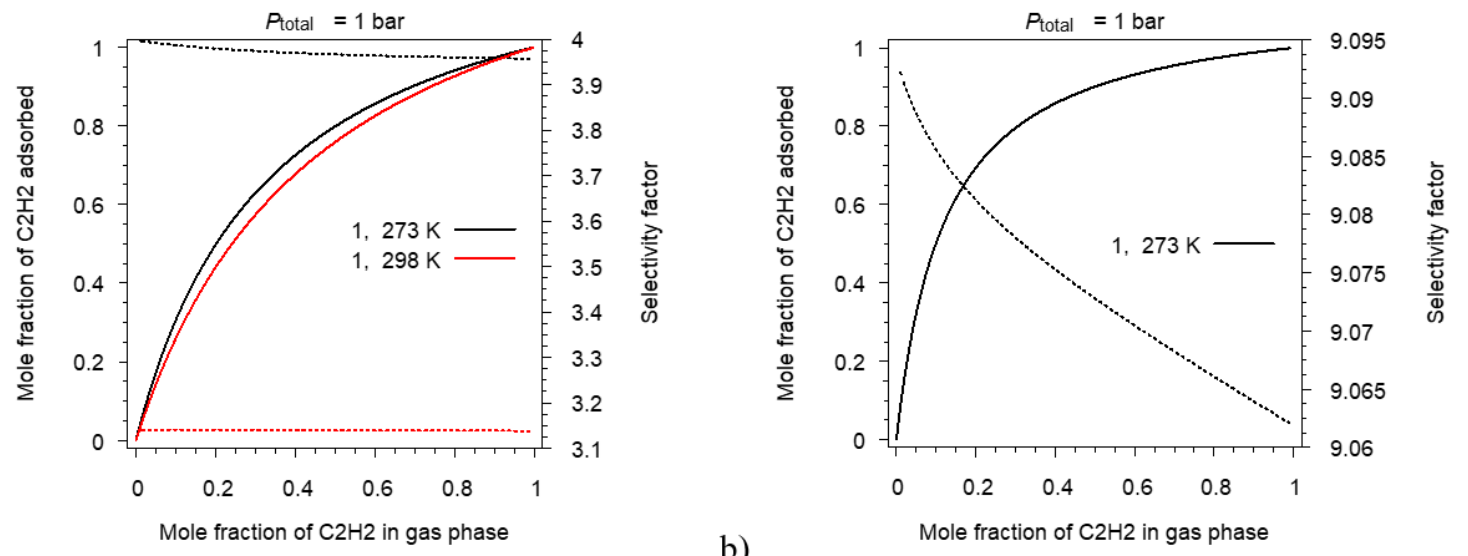

a)
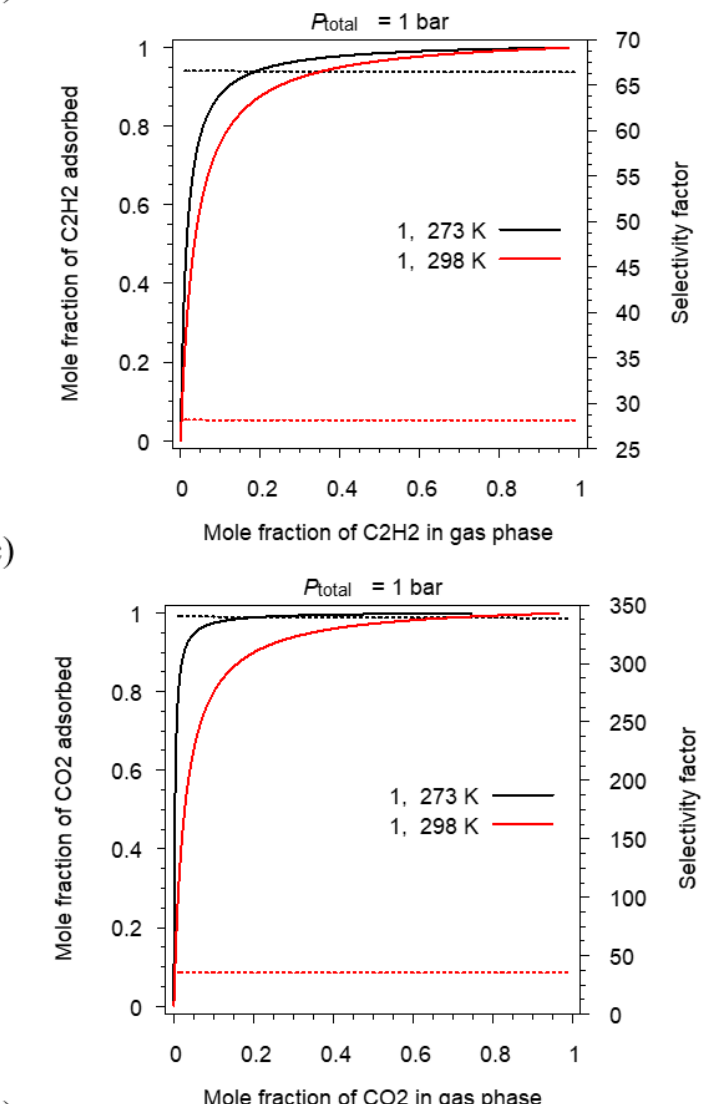

d)
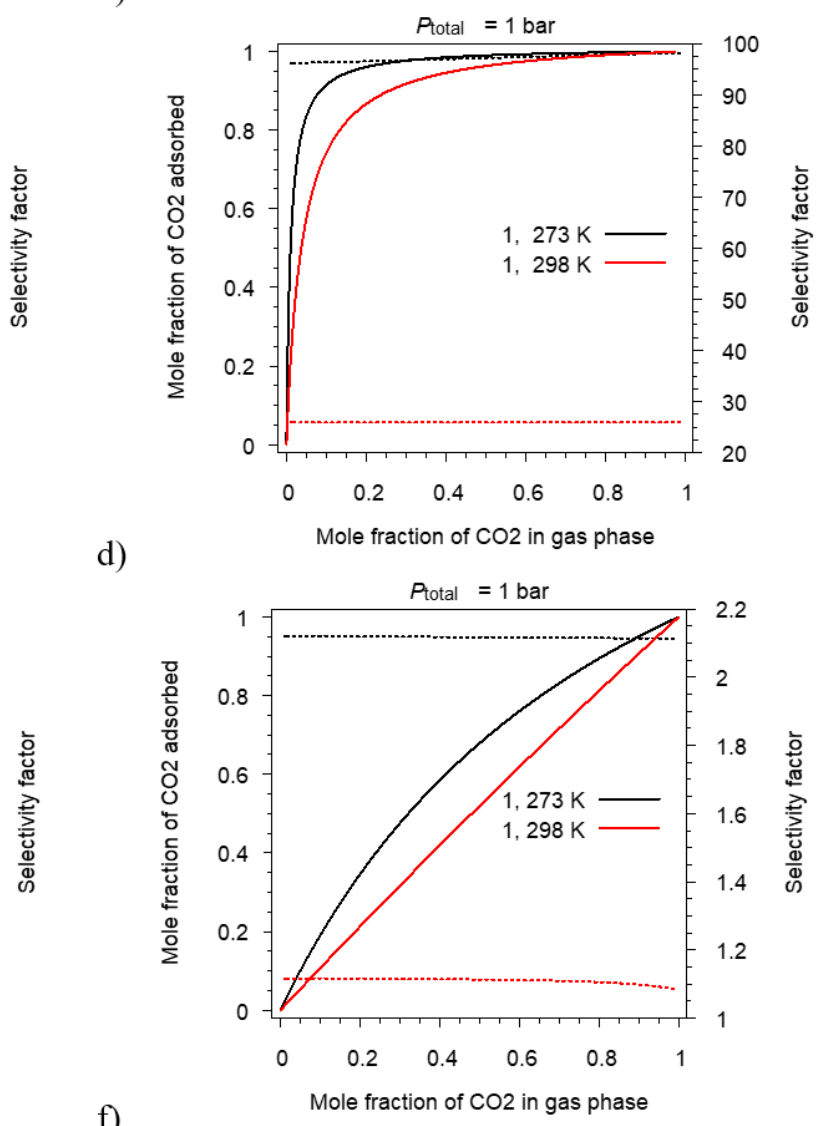

e)

Fig. S13. Prediction of adsorption equilibrium by IAST (solid lines) and dependence of selectivity factors (dashed lines) for binary gas mixtures adsorption on 1: a) $\mathrm{C}_{2} \mathrm{H}_{2} / \mathrm{C}_{2} \mathrm{H}_{4}$; b) $\mathrm{C}_{2} \mathrm{H}_{2} / \mathrm{C}_{2} \mathrm{H}_{6}$; c) $\mathrm{C}_{2} \mathrm{H}_{2} / \mathrm{CH}_{4} ;$ d) $\mathrm{CO}_{2} / \mathrm{CH}_{4}$; e) $\mathrm{CO}_{2} / \mathrm{N}_{2}$, f) $\mathrm{CO}_{2} / \mathrm{C}_{2} \mathrm{H}_{2}$. 


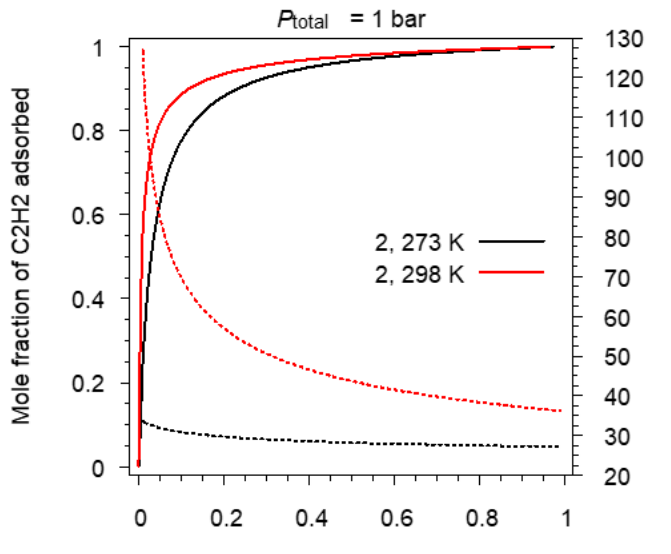

a)

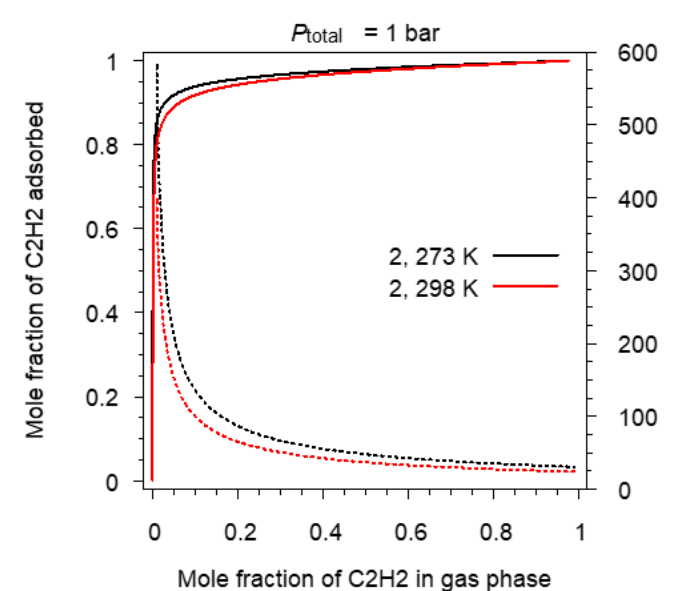

c)

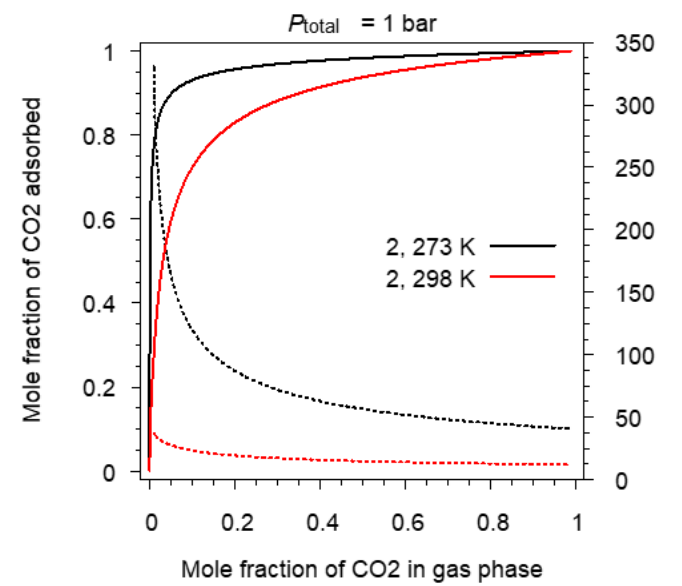

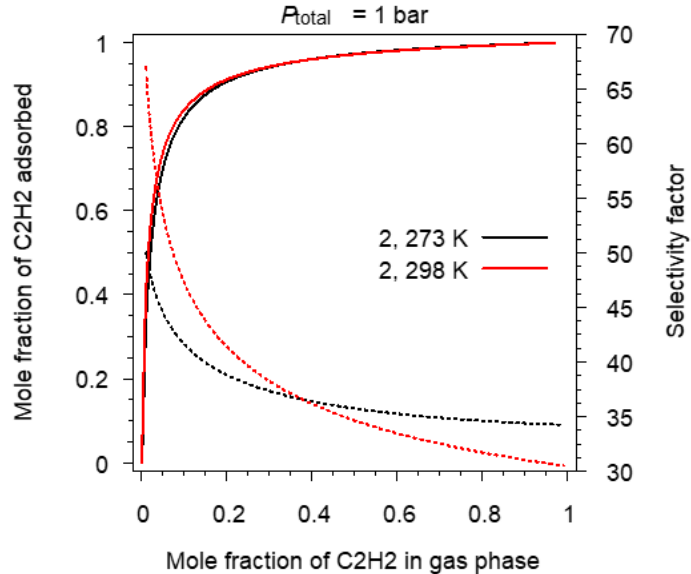

b)

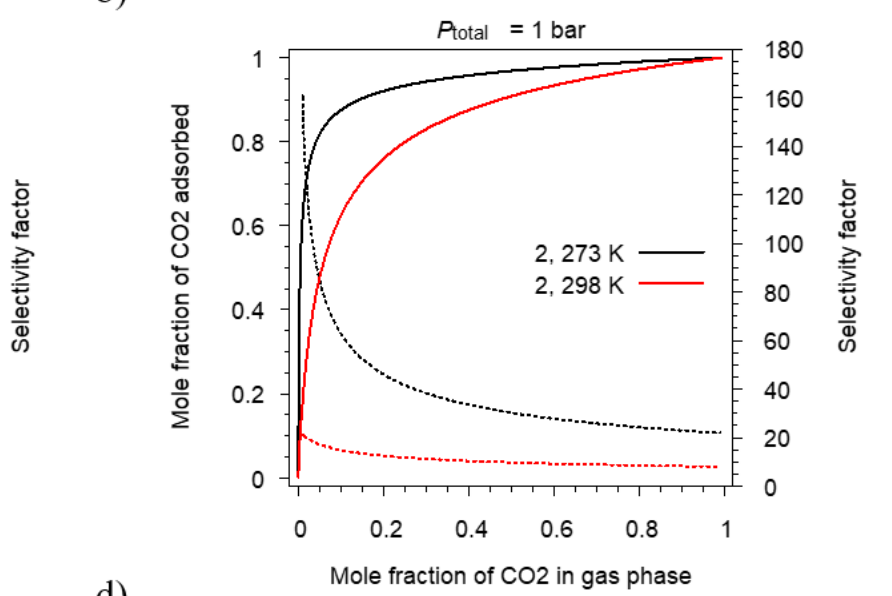

d)

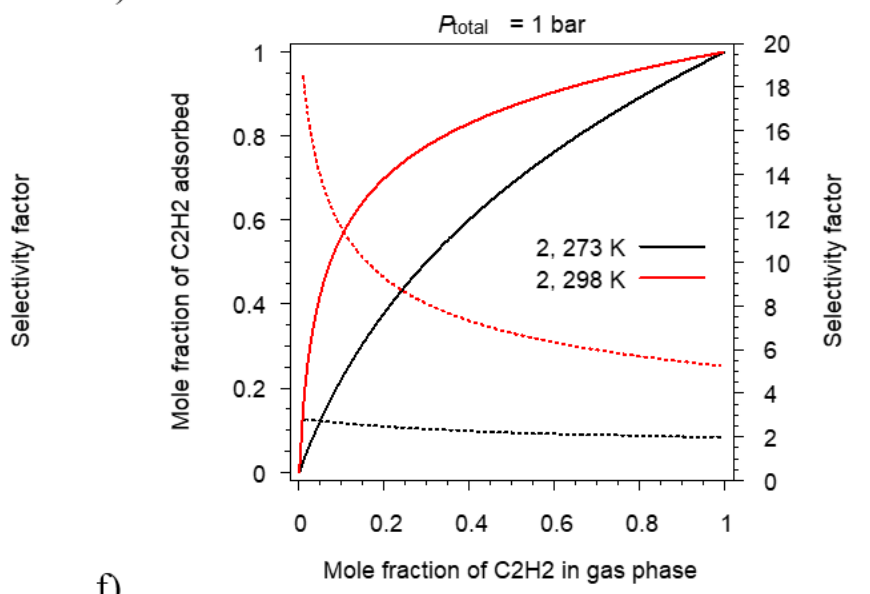

f)

\section{空}

Fig. S14. Prediction of adsorption equilibrium by IAST (solid lines) and dependence of selectivity factors (dashed lines) for binary gas mixtures adsorption on 2: a) $\mathrm{C}_{2} \mathrm{H}_{2} / \mathrm{C}_{2} \mathrm{H}_{4} ;$ b) $\mathrm{C}_{2} \mathrm{H}_{2} / \mathrm{C}_{2} \mathrm{H}_{6}$; c) $\mathrm{C}_{2} \mathrm{H}_{2} / \mathrm{CH}_{4}$; d) $\mathrm{CO}_{2} / \mathrm{CH}_{4}$; e) $\mathrm{CO}_{2} / \mathrm{N}_{2}$, f) $\mathrm{C}_{2} \mathrm{H}_{2} / \mathrm{CO}_{2}$. 
Table S7. Comparison of $\mathrm{C}_{2} \mathrm{H}_{2}$ uptake at 0.01 and 1 bar and $1 / 99 \mathrm{C}_{2} \mathrm{H}_{2} / \mathrm{C}_{2} \mathrm{H}_{4}$ selectivity in representative MOFs.

\begin{tabular}{|c|c|c|c|c|c|}
\hline & $\begin{array}{c}\mathrm{C}_{2} \mathrm{H}_{2} \\
\text { capacity at } \\
0.01 \mathrm{bar} \\
\mathrm{mmol} / \mathrm{g}\end{array}$ & $\begin{array}{c}\mathrm{C}_{2} \mathrm{H}_{2} \\
\text { capacity at } \\
1 \mathrm{bar}, \\
\mathrm{mmol} / \mathrm{g}\end{array}$ & Water-stability & $\begin{array}{c}\text { Selectivity } \\
\text { IAST }\end{array}$ & Ref \\
\hline NCU-100 & 0.73 & 4.57 & $\begin{array}{c}+ \text { (according to } \\
\text { PXRD) }\end{array}$ & 7291 & S1 \\
\hline $\begin{array}{c}\text { UTSA-200 } \\
\text { (SIFSIX-14-Cu-i) }\end{array}$ & 1.82 & 3.65 & - & $6320^{a}$ & S2 \\
\hline UTSA-300 & 0.04 & 3.08 & - & 27 & S3 \\
\hline SIFSIX-2-Cu-i & 1.88 & 4.02 & - & 44.54 & S4 \\
\hline SIFSIX-1-Cu & 1.02 & 8.5 & - & 10.63 & S4 \\
\hline SIFSIX-3-Zn & 0.50 & 3.64 & - & 8.82 & S4 \\
\hline SIFSIX-3-Ni & 0.23 & 3.30 & - & 5.03 & S5 \\
\hline ELM-12 & 0.43 & 2.56 & - & 14.8 & S6 \\
\hline NKMOF-1-Ni & 1.69 & 2.72 & $\begin{array}{l}+ \text { (according to } \\
\text { PXRD and } \\
\text { adsorption test) }\end{array}$ & 1272.6 & S7 \\
\hline UTSA-100 & 0.92 & 4.27 & - & 10.72 & S8 \\
\hline ZU-33 & 1.94 & 3.78 & - & $1100^{a}$ & S9 \\
\hline ZU-32 & 1.54 & 3.96 & - & 67 & S9 \\
\hline 1 & 0.01 & 0.326 & $\begin{array}{l}+ \text { (according to } \\
\text { PXRD and } \\
\text { adsorption test) }\end{array}$ & 3 & $\begin{array}{l}\text { This } \\
\text { work }\end{array}$ \\
\hline 2 & 0.33 & 2.7 & $\begin{array}{l}+ \text { (according to } \\
\text { PXRD and } \\
\text { adsorption test) }\end{array}$ & 127 & $\begin{array}{l}\text { This } \\
\text { work }\end{array}$ \\
\hline TIFSIX-2-Cu-i & 1.7 & 4.1 & - & 55 & $\mathrm{~S} 10$ \\
\hline TIFSIX-4-Cu-i & 0.45 & 4.3 & - & 11 & $\mathrm{~S} 10$ \\
\hline NOTT-300 & 0.25 & $6.34^{\mathrm{b}}$ & $\begin{array}{l}+ \text { (according to } \\
\text { PXRD and } \\
\text { adsorption test) }\end{array}$ & 2.17 & S11 \\
\hline ZU-62-Ni & 0.3 & 30.1 & $\begin{array}{l}+ \text { (according to } \\
\text { PXRD and } \\
\text { adsorption test) }\end{array}$ & 37.2 & S12 \\
\hline M'MOF-3 & 0.28 & 1.9 & - & 24.03 & $\mathrm{~S} 13$ \\
\hline Fe-MOF-74 & 1.26 & $6.8^{\mathrm{c}}$ & - & 2.08 & S14 \\
\hline ZU-62 & 1.1 & 3.53 & $\begin{array}{l}+ \text { (according to } \\
\text { PXRD and } \\
\text { adsorption test) }\end{array}$ & 24 & S15 \\
\hline ZU-12-Ni & 1.15 & 4.02 & - & 22.7 & S16 \\
\hline
\end{tabular}

${ }^{\mathrm{a}}$ only for the qualitative comparison

${ }^{b}$ at temperature of $293 \mathrm{~K}$

${ }^{\mathrm{c}}$ at temperature of $318 \mathrm{~K}$ 


\section{DFT calculations}

All density functional theory calculations were carried out under periodic boundary conditions with the Vienna Ab initio Simulation Package (VASP 5.4.4) [S17] using the projector-augmented wave (PAW) method [S18]. We employed PBE PAW potentials (v. 5.4) for all elements and exchange-correlation functionals. For the Sc, O, N, and C atoms, 3s $3 \mathrm{p} 3 \mathrm{~d} 4 \mathrm{~s}$, $2 \mathrm{~s} 2 \mathrm{p}, 2 \mathrm{~s} 2 \mathrm{p}$, and $2 \mathrm{~s} 2 \mathrm{p}$ electrons, respectively, were considered as valence electrons, while for the $\mathrm{H}$ atoms, the $1 \mathrm{~s}$ electron was explicitly treated.

Structural relaxations were performed using the PBE functional [S19] with D3(BJ) dispersion correction [S20, S21] and a plane-wave kinetic-energy cutoff of $400 \mathrm{eV}$. The final energies of species under study were calculated by the recently developed SCAN $+r \mathrm{VV} 10$ method [S22], which shows outstanding performance in molecular adsorption studies, in combination with a plane-wave kinetic-energy cutoff of $500 \mathrm{eV}$ on top of PBE-D3(BJ) structures.

The electronic (ionic) convergence criterion was set to $10^{-6}\left(10^{-5}\right) \mathrm{eV}$ and Gaussian smearing with a smearing width of $0.05 \mathrm{eV}$ was used throughout the computations. Only the $\Gamma$ point was used for sampling the Brillouin zone during structural relaxations, whereas for singlepoint calculations the Brillouin zone was integrated using $\Gamma$-centered grids with a consistent spacing density employing KSPACING parameter set to $0.5 \AA^{-1}$.

The difference between the energy of adsorbed system $\left(E_{\text {guest }} @\right.$ host $)$ and the sum of energies of the empty metal-organic framework $\left(E_{\text {host }}\right)$ and non-coordinated guest molecules $\left(n \times E_{\text {guest }}\right.$, where $n$ equal to the number of adsorbed molecules) was used to compute the adsorption energies $(\Delta E)$ according to expression:

$$
\Delta E=E_{\text {guest }} @_{\text {host }}-\left(n \times E_{\text {guest }}+E_{\text {host }}\right) .
$$

Hirshfeld partial charges [S23] were calculated at the PBE-MBD@rsSCS level of theory [S24-S26] using the same settings as for the SCAN+rVV10 single-point calculations. 

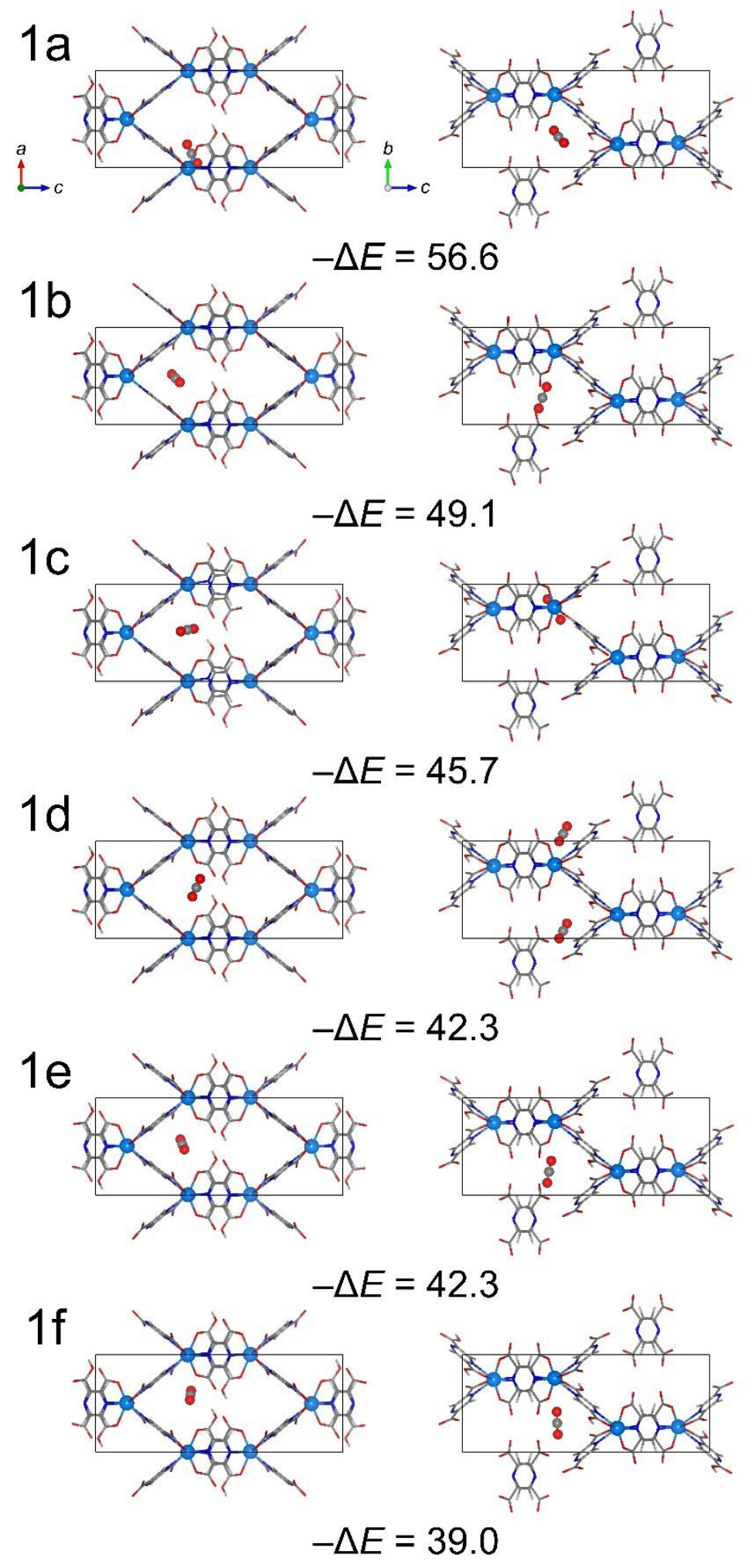

Figure S15. Calculated structures and adsorption energies $\left(\Delta E, \mathrm{~kJ}^{\circ} \cdot \mathrm{mol}^{-1}\right)$ of one $\mathrm{CO}_{2}$ molecule at representative adsorption sites inside 1 pores. 

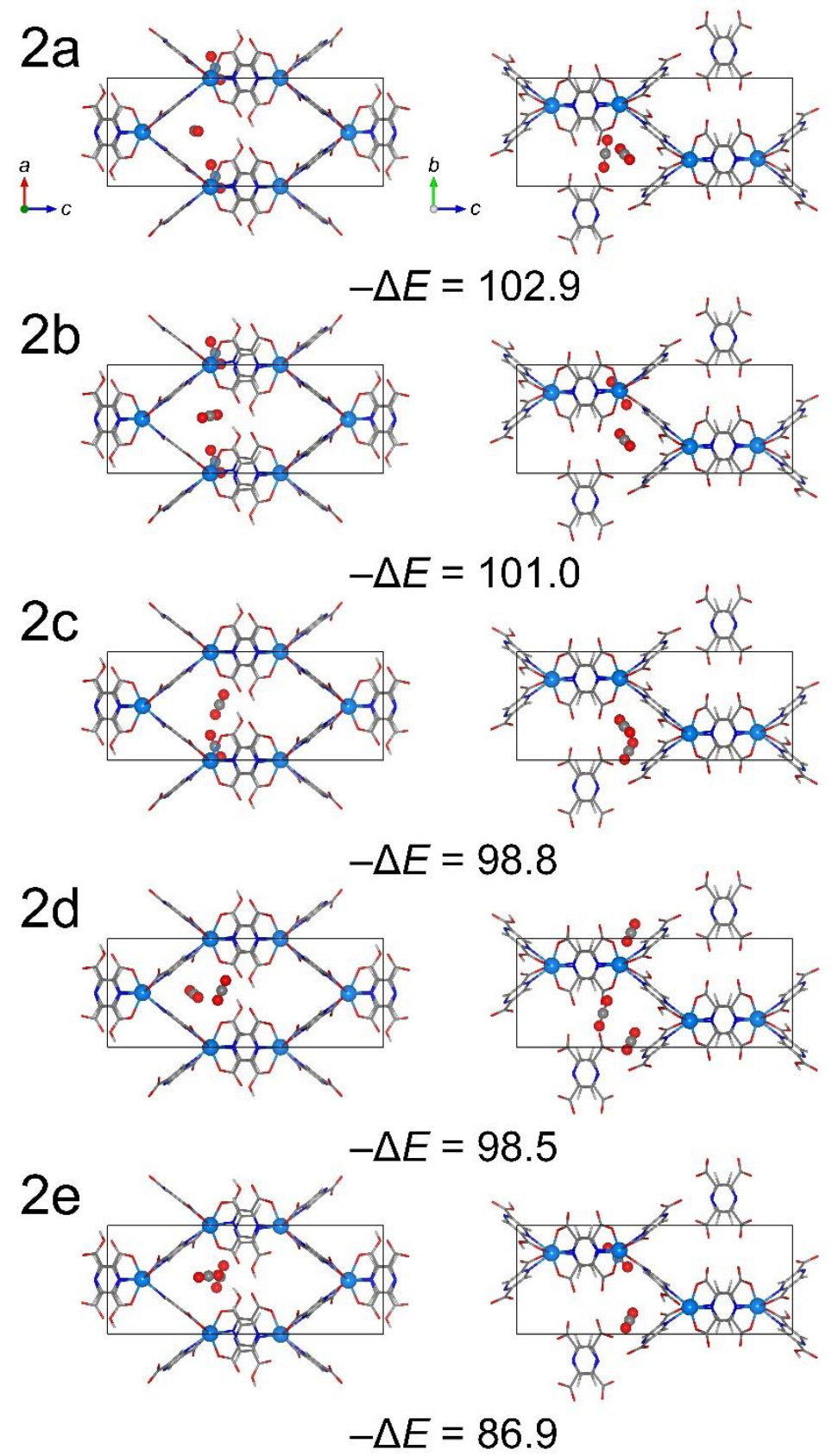

Figure S16. Calculated structures and adsorption energies $\left(\Delta E, \mathrm{~kJ} \cdot \mathrm{mol}^{-1}\right)$ of two $\mathrm{CO}_{2}$ molecules at representative adsorption sites inside 1 pores. 

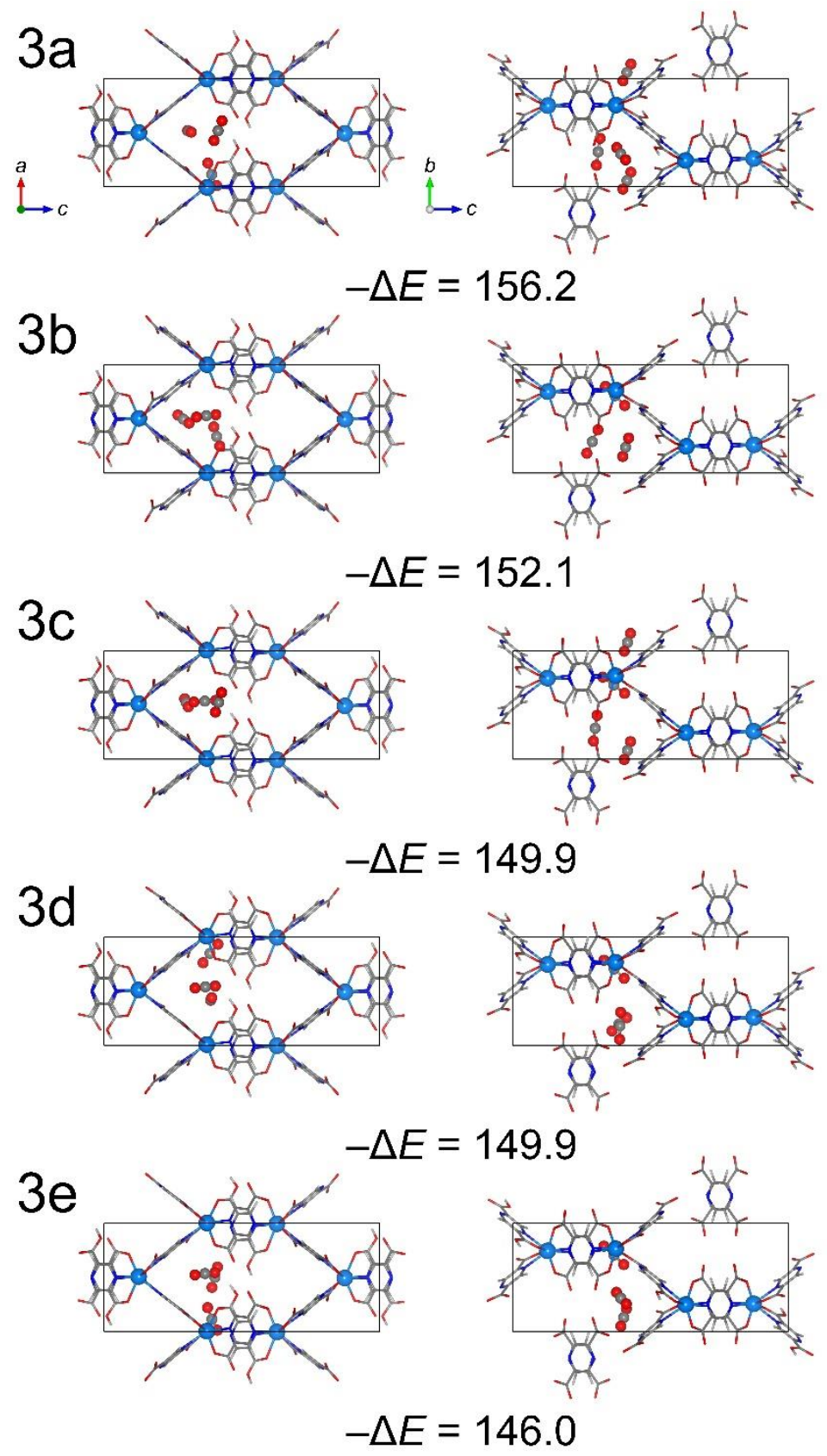

Figure S17. Calculated structures and adsorption energies $\left(\Delta E, \mathrm{~kJ} \cdot \mathrm{mol}^{-1}\right)$ of three $\mathrm{CO}_{2}$ molecules at representative adsorption sites inside 1 pores. 

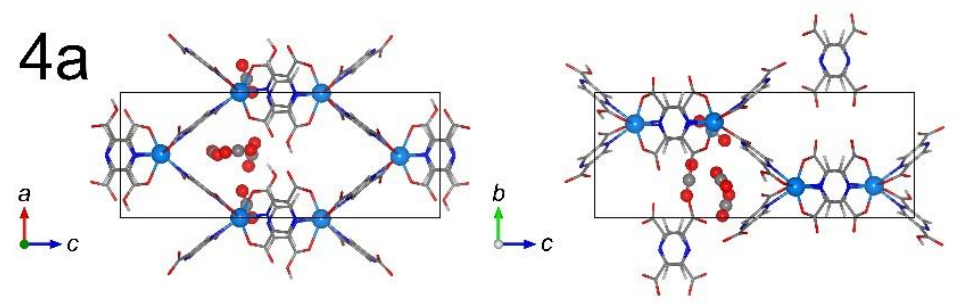

$-\Delta E=209.1$

$4 \mathrm{~b}$
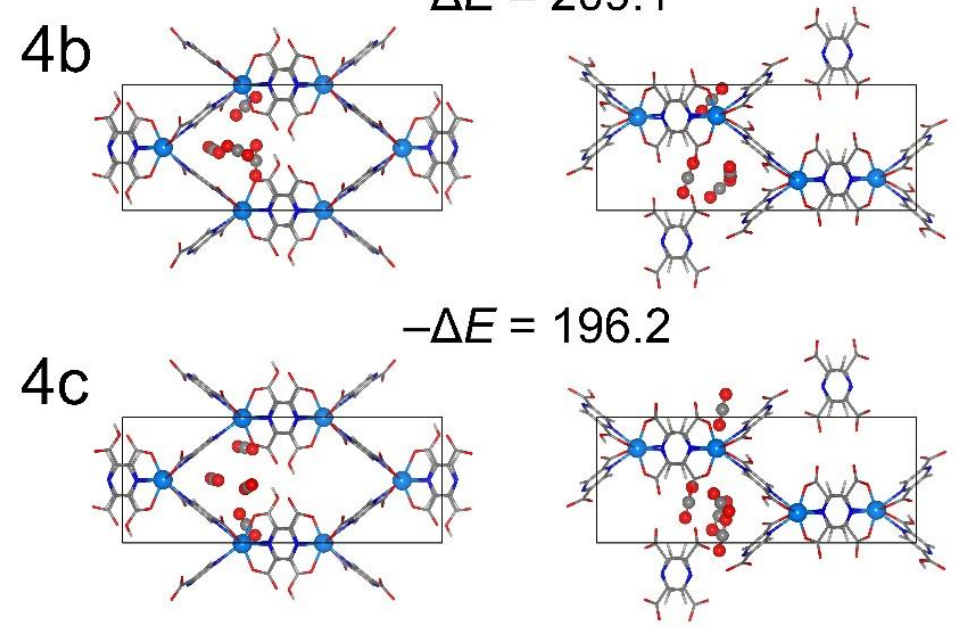

$-\Delta E=188.8$

Figure S18. Calculated structures and adsorption energies $\left(\Delta E, \mathrm{~kJ} \cdot \mathrm{mol}^{-1}\right)$ of four $\mathrm{CO}_{2}$ molecules at representative adsorption sites inside 1 pores. 


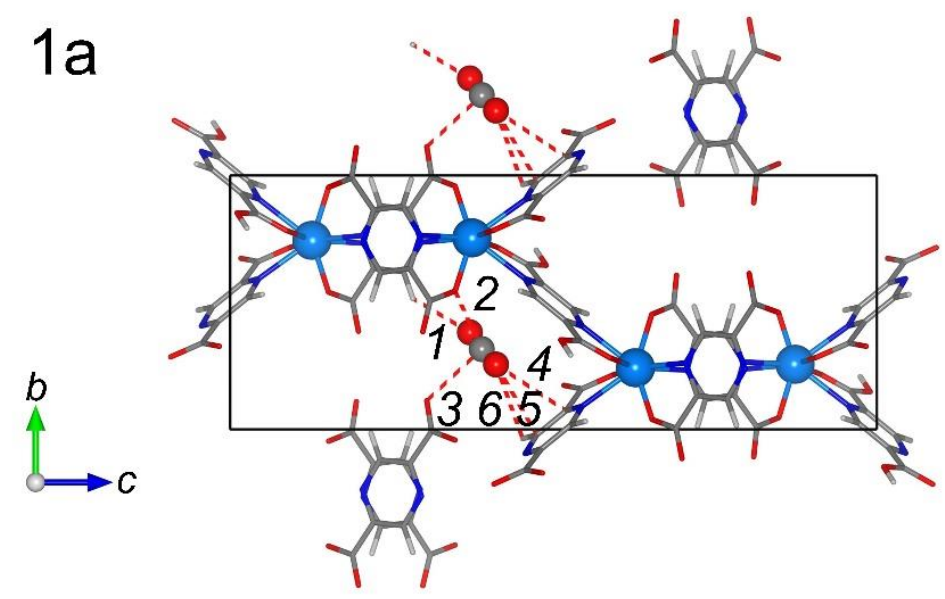

\begin{tabular}{|lrrr|}
\hline Site 1a & \multicolumn{3}{c|}{$\mathbf{C O}_{2} @ 1$} \\
\hline Contact & $r, \AA$ & $q_{\text {guest }},|\mathrm{e}|$ & $q_{\text {host }},|\mathrm{e}|$ \\
$\mathbf{1}(\mathbf{O} \cdots \mathrm{H})$ & 2.54 & -0.13 & 0.06 \\
$\mathbf{2}(\mathbf{C} \cdots \mathbf{O})$ & 2.95 & 0.28 & -0.28 \\
$\mathbf{3}(\mathbf{C} \cdots \mathbf{O})$ & 2.83 & 0.28 & -0.24 \\
$\mathbf{4}(\mathbf{O} \cdots \mathbf{C})$ & 3.14 & -0.14 & 0.06 \\
$\mathbf{5}(\mathbf{O} \cdots \mathbf{C})$ & 3.07 & -0.14 & 0.04 \\
$\mathbf{6}(\mathbf{O} \cdots \mathbf{H})$ & 3.33 & -0.14 & 0.06
\end{tabular}

Figure S19. Calculated interatomic distances $(\AA)$ of the shortest $\mathbf{X}_{\text {guest }} \cdots \mathbf{Y}_{\text {host }}$ contacts and the corresponding Hirshfeld partial charges for $\mathrm{CO}_{2}$ molecule adsorbed on the inner surface of 1 at site $1 a$.

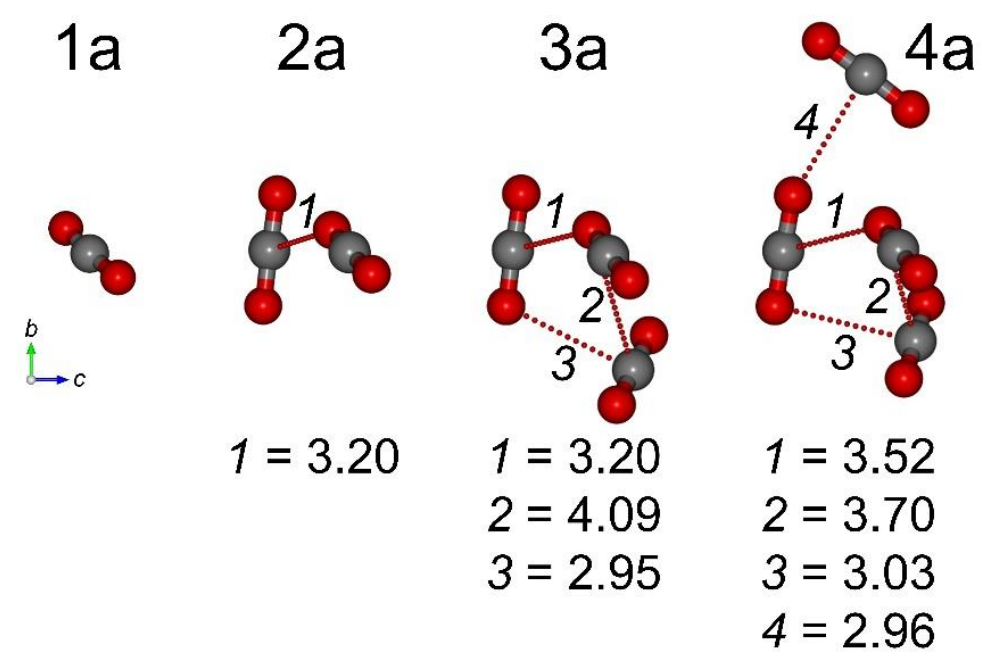

Figure S20. Calculated interatomic distances $(\AA)$ of the shortest $\mathrm{C} \cdots \mathrm{O}$ contacts for $\left(\mathrm{CO}_{2}\right)_{n}$ molecules, where $n=1-4$, adsorbed on the inner surface of 1 at the most energetically preferred sites 1a-4a. 

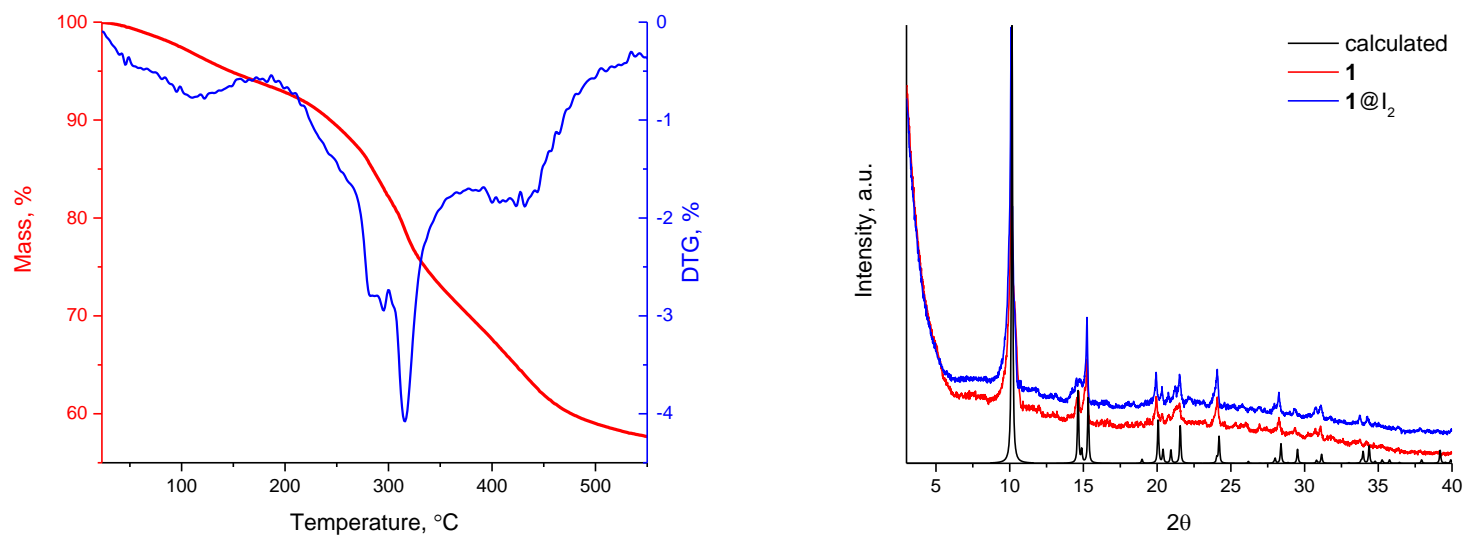

Fig. S21. TG curves and PXRD patterns for $1 \cdot 0.4 \mathrm{I}_{2} \cdot 1.5 \mathrm{H}_{2} \mathrm{O}$
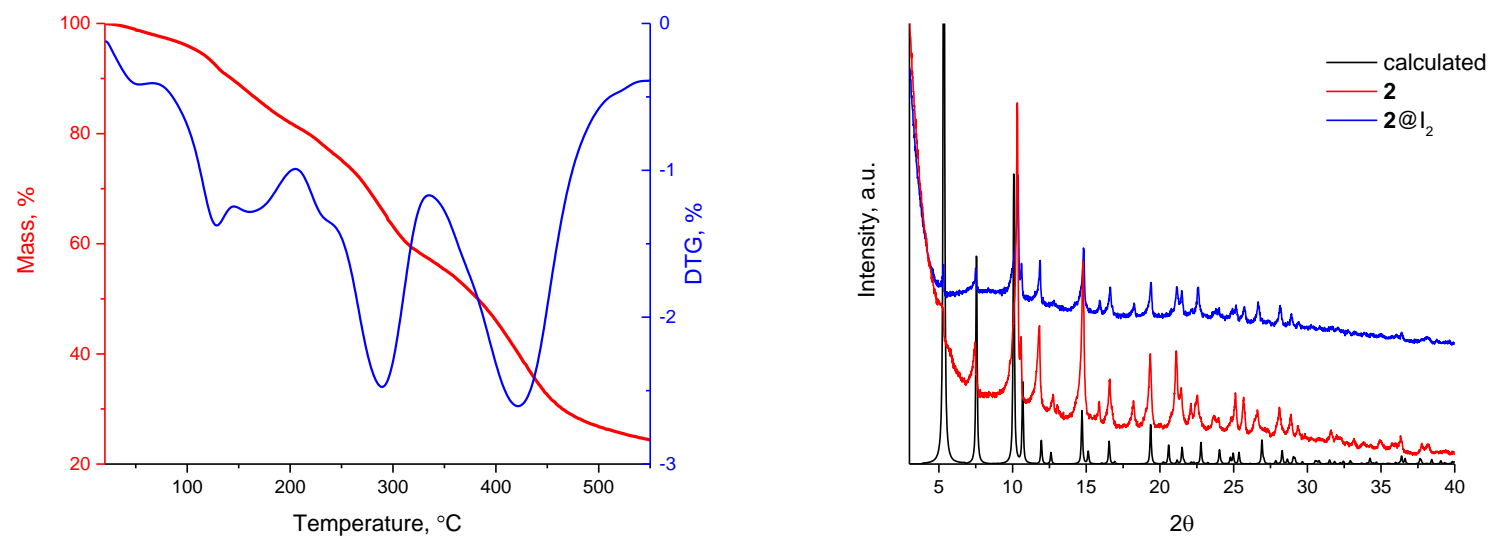

Fig. S22. TG curves and PXRD patterns for $2 \cdot \mathbf{I}_{2} \cdot 2 \mathrm{H}_{2} \mathrm{O}$
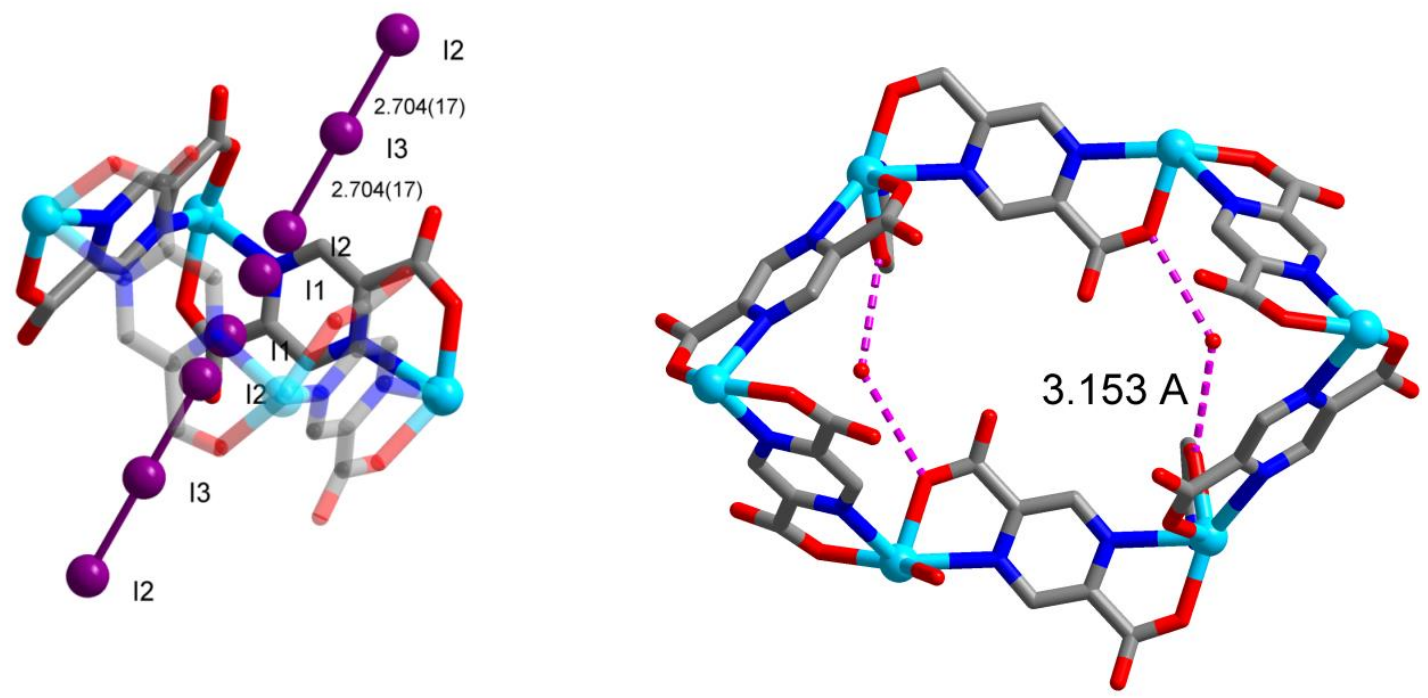

Fig. S23. Positions of iodine atoms and water molecules from single crystal XRD for $\mathbf{2} \cdot \mathbf{I}_{2} \cdot \mathbf{2} \mathrm{H}_{2} \mathrm{O}$. 
S1) J. Wang, Y. Zhang, P. Zhang, J. Hu, R.-B. Lin, Q. Deng, Z. Zeng, H. Xing, S. Deng, B. Chen, J. Am. Chem. Soc. 2020, 142, 9744-9751;

S2) B. Li, X. Cui, D. O'Nolan, H.-M. Wen, M. Jiang, R. Krishna, H. Wu, R.-B. Lin, Y.-S. Chen, D. Yuan, H. Xing, W. Zhou, Q. Ren, G. Qian, M. J. Zaworotko, B. Chen, Adv. Mater. 2017, 29, 1704210;

S3) R.-B. Lin, L. L, H. Wu, H. Arman, B. Li, R.-G. Lin, W. Zhou, B. Chen, J. Am. Chem. Soc. 2017, 139, 8022;

S4) X. Cui, K. Chen, H. Xing, Q. Yang, R. Krishna, Z. Bao, H. Wu, W. Zhou, X. Dong, Y. Han, B. Li, Q. Ren, M. J. Zaworotko, B. Chen, Science, 2016, 353, 141;

S5) L. Yang, X. Cui, Q. Yang, S. Qian, H. Wu, Z. Bao, Z. Zhang, Q. Ren, W. Zhou, B. Chen, H. Xing, Adv. Mater, 2018, 30, 1705374;

S6) L. Li, R-B. Lin, R. Krishna, X. Wang, B. Li, H. Wu, J. Li, W. Zhou, B. Chen, J. Mater. Chem. A 2017, 5, 18984;

S7) Y.-L. Peng, T. Pham, P. Li, T. Wang, Y. Chen, K.-J. Chen, K. A. Forrest, B. Space, P. Cheng, M. J. Zaworotko, Z. Zhang, Angew. Chem. Int. Ed, 2018, 57, 10971;

S8) T.-L. Hu, H. Wang, B. Li, R. Krishna, H. Wu, W. Zhou, Y. Zhao, Y. Han, X. Wang, W. Zhu, Z. Yao, S. Xiang, B. Chen, Nat. Commun. 2015, 6, 7328;

S9) Z. Zhang, X. Cui, L. Yang, J. Cui, Z. Bao, Q. Yang, H. Xing, Ind. Eng. Chem. Res, 2018, 57, 7266;

S10) A. Bajpai, D. O'Nolan, D. G. Madden, K.-J. Chen, T. Pham, A. Kumar, M. Lusi, J. J. Perry, IV, B. Space, M. J. Zaworotko, Chem. Commun., 2017, 53, 11592

S11) S. Yang, A. J. Ramirez-Cuesta, R. Newby, V. Garcia-Sakai, P. Manuel, S. K. Callear, S. I. Campbell, C. C. Tang, M. Schröder, Nat. Chem. 2015, 7, 121;

S12) L. Yang, A. Jin , L. Ge, X. Cui, H. Xing, Chem. Commun., 2019, 55, 5001;

S13) S.-C. Xiang, Z. Zhang, C.-G. Zhao, K. Hong, X. Zhao, D.-R. Ding, M.-H. Xie, C.-D. Wu, M. C. Das, R. Gill, K. M. Thomas, B. Chen, Nat. Commun, 2011, 2, 204;

S14) E. D. Bloch, W. L. Queen, R. Krishna, J. M. Zadrozny, C. M. Brown, J. R. Long, Science, 2012, 335, 1606;

S15) L. Yang, X. Cui, Z. Zhang, Q. Yang, Z. Bao, Q. Ren, H. Xing, Angew. Chem. Int. Ed, 2018, 57, 13145;

S16) M. Jiang, X. Cui, L. Yang, Q. Yang, Z. Zhang, Y. Yang, H. Xing, Chem. Eng. J, 2018, 352, 803 ;

S17) G. Kresse, J. Furthmüller, Phys. Rev. B 1996, 54, 11169;

S18) G. Kresse, D. Joubert, Phys. Rev. B 1999, 59, 1758;

S19) J. P. Perdew, K Burke, M. Ernzerhof, Phys Rev Lett. 1996, 77, 3865; 
S20) S. Grimme, J. Antony, S. Ehrlich, H. Krieg, J. Chem. Phys. 2010, 132, 154104;

S21) S. Grimme, S. Ehrlich, L. Goerigk, J. Comput. Chem. 2011, 32, 1456;

S22) H. Peng, Z.-H. Yang, J. P. Perdew, J. Sun, Phys. Rev. X 2016, 6, 041005;

S23) P. Bultinck, J. Chem. Phys. 2007, 126, 144111;

S24) A. Tkatchenko, R. A. DiStasio, Jr., R. Car, M. Scheffler, Phys. Rev. Lett. 2012, 108, 236402;

S25) J. Iles-Smith, A. G. Dijkstra, N. Lambert, A. Nazir, J. Chem. Phys. 2014, 140, 018A508;

S26) T. Bučko, S. Lebègue, T. Gould, J. G Ángyán, J. Phys.: Condens. Matter 2016, 28, 045201; 